\title{
Spectral Estimates and Non-Selfadjoint Perturbations of Spheroidal Wave Operators
}

\author{
Felix Finster and Harald Schmid
}

May 2004 / May 2005

\begin{abstract}
We derive a spectral representation for the oblate spheroidal wave operator which is holomorphic in the aspherical parameter $\Omega$ in a neighborhood of the real line. For real $\Omega$, estimates are derived for all eigenvalue gaps uniformly in $\Omega$.

The proof of the gap estimates is based on detailed estimates for complex solutions of the Riccati equation. The spectral representation for complex $\Omega$ is obtained using the theory of slightly non-selfadjoint perturbations.
\end{abstract}

\section{Introduction}

Recently an integral representation was derived for solutions of the scalar wave equation in the Kerr black hole geometry [4. This result relies crucially on a spectral representation for the oblate spheroidal wave operator for complex values of the aspherical parameter $\Omega$ (also referred to as "ellipticity parameter" or "semifocal distance"). In the present paper, this spectral representation is proved. The reason why this problem deserves to be worked out in a separate paper is that most of our methods apply in a much more general context. Namely, the core of the paper is to derive estimates for the eigenvalue gaps $\lambda_{n+1}-\lambda_{n}$ for real $\Omega$, which are uniform in $\Omega$ and $n$. To this end, we need to control the eigenvalues and the behavior of the wave functions in detail. Our method is based on invariant region estimates for the complex Riccati equation and applies to general Sturm-Liouville or onedimensional Schrödinger problems. In particular, it gives refined error estimates for WKB approximations. We regard the spheroidal wave equation as a model problem for working out these estimates.

Despite the vast literature on spectral estimates for the Schrödinger equation (see e.g. 12 and the references therein), gap estimates are rarely found in the standard literature. Most papers are concerned with the two lowest eigenvalues [9, 14, or they apply in special situations like for a a nearly constant potential [10]. Probably, this is because gap estimates depend sensitively on the detailed form of the potential (as one sees in the example of a double-well potential), making it difficult to get general results. Our method requires that the potential is piecewise monotone and that we have good control of its derivatives.

We now introduce our problem and state our results. The spheroidal wave equation is the eigenvalue equation for the spheroidal wave operator, a linear elliptic operator with smooth coefficients on the unit sphere $S^{2}$. Since the spheroidal wave operator is axisymmetric, we can choose angular variables $\vartheta \in(0, \pi)$ and $\varphi \in[0,2 \pi)$ (with $\vartheta$ the angle to the axis of symmetry) and separate out the $\varphi$-dependence with the plane wave ansatz 
$\phi(\vartheta, \varphi)=e^{i k \varphi} \Theta(\vartheta), k \in \mathbb{Z}$. After this separation, the spheroidal wave equation takes the form

$$
\mathcal{A} \Theta=\lambda \Theta
$$

where $\mathcal{A}$ is the linear differential operator of second order

$$
\mathcal{A}=-\frac{d}{d \cos \vartheta} \sin ^{2} \vartheta \frac{d}{d \cos \vartheta}+\frac{1}{\sin ^{2} \vartheta}\left(\Omega \sin ^{2} \vartheta+k\right)^{2}
$$

on the interval $\vartheta \in(0, \pi)$. Here $\Omega \in \mathbb{C}$ is the aspherical parameter. In the special case $\Omega=0$, the spheroidal wave operator simplifies to the spherical Laplacian, and the Legendre polynomials $P_{l}^{k}(\cos \vartheta)$ are explicit solutions to (1.1). We shall consider the spheroidal wave equation for fixed $k$, but for a variable complex parameter $\Omega$. The fact that the eigenfunction $\phi$ should be smooth at the poles $\vartheta=0, \pi$ of the sphere gives rise to the following boundary conditions,

$$
\begin{cases}\lim _{\vartheta \rightarrow 0, \pi} \Theta^{\prime}(\vartheta)=0 & \text { if } k=0 \\ \lim _{\vartheta \rightarrow 0, \pi} \Theta(\vartheta)=0 & \text { if } k \neq 0 .\end{cases}
$$

We consider $\mathcal{A}$ as an operator in the Hilbert space $\mathcal{H}=L^{2}((0, \pi), \sin \vartheta d \vartheta)$ with domain of definition given by those functions in $C^{2}(0, \pi)$ which satisfy the boundary conditions (1.3). Note that the potential in the spheroidal wave operator is in general complex,

$$
\operatorname{Im}\left(\frac{\left(\Omega \sin ^{2} \vartheta+k\right)^{2}}{\sin ^{2} \vartheta}\right)=2\left(\operatorname{Re} \Omega \sin ^{2} \vartheta+k\right) \operatorname{Im} \Omega
$$

and therefore $\mathcal{A}$ is symmetric only if $\Omega$ is real. In previous works, asymptotic expansions for individual eigenvalues are derived [5, 11], and it is shown numerically that eigenvalues can degenerate for non-real $\Omega$ [], but rigorous estimates or completeness statements are not given. Our main result is the following spectral representation for $\Omega$ in a neighborhood of the real line.

Theorem 1.1 For any $k \in \mathbb{Z}$ and $c>0$, we define the open set $U \subset \mathbb{C}$ by the condition

$$
|\operatorname{Im} \Omega|<\frac{c}{1+|\operatorname{Re} \Omega|} .
$$

Then there is a positive integer $N$ and a family of operators $Q_{n}(\Omega)$ on $\mathcal{H}$ defined for $n \in \mathbb{N} \cup\{0\}$ and $\Omega \in U$ with the following properties:

(i) The $Q_{n}$ are holomorphic in $\Omega$.

(ii) $Q_{0}$ is a projector on an $N$-dimensional invariant subspace of $\mathcal{A}$. For $n>0$, the $Q_{n}$ are projectors on one-dimensional eigenspaces of $\mathcal{A}$ with corresponding eigenvalues $\lambda_{n}(\Omega)$. These eigenvalues satisfy a bound of the form

$$
\left|\lambda_{n}(\Omega)\right| \leq C(n)(1+|\Omega|)
$$

for suitable constants $C(n)$. Furthermore, there is a parameter $\varepsilon>0$ such that for all $n \in \mathbb{N}$ and $\Omega \in U$,

$$
\left|\lambda_{n}(\Omega)\right| \geq n \varepsilon .
$$


(iii) The $Q_{n}$ are complete, i.e.

$$
\sum_{k=0}^{\infty} Q_{n}=\mathbb{1}
$$

with strong convergence of the series.

(iv) The $Q_{n}$ are uniformly bounded, i.e. for all $n \in \mathbb{N} \cup\{0\}$,

$$
\left\|Q_{n}\right\| \leq c_{1}
$$

with $c_{1}$ independent of $\Omega$ and $k$.

If $c$ is sufficiently small, $c<\delta$, or the real part of $\Omega$ is sufficiently large, $|\operatorname{Re} \Omega|>C(c)$, one can choose $N=1$, i.e. $\mathcal{A}$ has a purely discrete spectrum consisting of simple eigenvalues.

To avoid misunderstandings, we point out that by a "projector on an invariant subspace of $\mathcal{A}$ " we mean an operator $Q$ which is idempotent and commutes with $\mathcal{A}$. But $Q$ will in general not be symmetric.

In our proof we shall treat the imaginary part of the potential (1.4) as a slightly nonselfadjoint perturbation in the spirit of [8, V.4.5], see also [2, Chapter 12]. For this method to be applicable, we need good control of the eigenvalues of the corresponding selfadjoint problem. Our starting point is the following spectral decomposition of $\mathcal{A}$ in the case of real $\Omega$.

Theorem 1.2 For any $k \in \mathbb{Z}$ and $\Omega \in \mathbb{R}$, the operator $\mathcal{A}$ has a unique selfadjoint extension compatible with the boundary conditions (1.3). This extension, which we again denote by $\mathcal{A}$, is a positive operator with compact resolvent and simple eigenvalues. It is invariant on the even and odd parity subspaces $\mathcal{H}^{ \pm}$defined by

$$
\mathcal{H}=\mathcal{H}^{+} \oplus \mathcal{H}^{-} \quad \text { with } \quad \mathcal{H}^{ \pm}=\{\phi \in \mathcal{H} \text { with } \phi(\pi-\vartheta)= \pm \phi(\vartheta)\} .
$$

We denote the eigenvalues of $\mathcal{A}$ restricted to $\mathcal{H}^{ \pm}$by $\lambda_{n}^{ \pm}$and count them with multiplicities,

$$
0 \leq \lambda_{1}^{ \pm}<\lambda_{2}^{ \pm}<\lambda_{3}^{ \pm}<\cdots
$$

Using abstract methods (see [8, Theorem 3.9, VII.3.5]), one could show that each eigenvalue $\lambda_{n}^{ \pm}(\Omega)$ has a holomorphic continuation to a neighborhood of the real axis. However, as pointed out in [8, Remark 3.9, VII.3.5], this neighborhood will depend on $n$, making it impossible construct a neighborhood in which all the $\lambda_{n}^{ \pm}(\Omega)$ exist. Therefore, abstract methods only seem to give results which are much weaker than Theorem 1.1. where the whole spectral decomposition is shown to have a holomorphic continuation to a neighborhood of the real axis. Furthermore, we point out that the parameter $c$ in the statement of Theorem 1.1 can be chosen arbitrarily large. Therefore, the holomorphic family of operators $Q_{n}(\Omega)$ is not only defined in a small neighborhood of the real axis, but in a strip (1.5) which can enclose any bounded subset of the complex plane. The key for getting this strong result are the following gap estimates uniform in $n$ and $\Omega$.

Theorem 1.3 For any $k \in \mathbb{Z}$ and $\gamma>0$, there is a positive integer $N$ such that

$$
\lambda_{n+1}^{ \pm}-\lambda_{n}^{ \pm}>\gamma \quad \text { for all } n \geq N \text { and } \Omega \in \mathbb{R} .
$$

If $\gamma$ is sufficiently small or $|\Omega|$ is sufficiently large, one can choose $N=1$. 
The paper is organized as follows. In Section 2 we prove Theorem 1.2 and reduce Theorem 1.3 to gap estimates for a self-adjoint Sturm-Liouville operator on the interval $\vartheta \in$ $\left(0, \frac{\pi}{2}\right]$ with suitable boundary conditions. In Sections 2 6 we introduce the complex Riccati equation and develop general techniques for analyzing its solutions. In Section 7 and 8 we apply these techniques to the spheroidal wave operator and prove Theorem 1.3. Finally, in Section 8 we use perturbative methods to proof Theorem 1.1

\section{Basic Considerations}

Until the end of Section 17 we will consider the spheroidal wave equation (1.1) for real $\Omega$. Using that (1.2) is invariant under the transformations $\Omega \rightarrow-\Omega$ and $k \rightarrow-k$, we can assume throughout that

$$
\Omega>0 \text {. }
$$

Let us derive a spectral representation of the spheroidal wave operator. One possible method would be to apply elliptic theory to the spheroidal wave operator on $S^{2}$ before separation of variables. After choosing a self-adjoint extension on the Hilbert space $L^{2}\left(S^{2}\right)$, one could apply the abstract spectral theorem, and projecting the resulting smooth eigenfunctions on the subspace for fixed $k$ would give the desired spectral decomposition for the ordinary differential operator (1.2). For clarity, we will in this paper restrict attention to ODE techniques. Thus we avoid elliptic theory and prefer to apply Sturm-Liouville theory. In the variable $u=\vartheta \in(0, \pi)$, the operator (1.2) can be written as

$$
\mathcal{A}=-\frac{1}{\sin u} \frac{d}{d u} \sin u \frac{d}{d u}+\frac{1}{\sin ^{2} u}\left(\Omega \sin ^{2} u+k\right)^{2} .
$$

In order to bring this operator to the standard Sturm-Liouville form, we introduce the function $Y$ by

$$
Y=\sqrt{\sin u} \Theta
$$

Then

$$
B Y=\lambda Y,
$$

where

$$
\begin{aligned}
B & =-\frac{1}{\sqrt{\sin u}} \frac{d}{d u} \sin u \frac{d}{d u} \frac{1}{\sqrt{\sin u}}+\frac{1}{\sin ^{2} u}\left(\Omega \sin ^{2} u+k\right)^{2} \\
& =-\frac{d^{2}}{d u^{2}}+\frac{1}{2} \frac{\cos ^{2} u}{\sin ^{2} u}-\sqrt{\sin u}\left(\frac{1}{\sqrt{\sin u}}\right)^{\prime \prime}+\frac{1}{\sin ^{2} u}\left(\Omega \sin ^{2} u+k\right)^{2} \\
& =-\frac{d^{2}}{d u^{2}}-\frac{1}{4} \frac{\cos ^{2} u}{\sin ^{2} u}-\frac{1}{2}+\frac{1}{\sin ^{2} u}\left(\Omega \sin ^{2} u+k\right)^{2} .
\end{aligned}
$$

Thus $Y$ satisfies the Sturm-Liouville equation

$$
\left(-\frac{d^{2}}{d u^{2}}+V\right) Y=0
$$

where $V$ is the potential

$$
V=\Omega^{2} \sin ^{2} u+\left(k^{2}-\frac{1}{4}\right) \frac{1}{\sin ^{2} u}-\mu
$$


with $\mu$ the constant

$$
\mu=\lambda-2 \Omega k+\frac{1}{4}
$$

The transformation (2.1) from $\Theta$ to $Y$ becomes a unitary transformation if the integration measure in the corresponding Hilbert spaces is transformed from $\sin u d u$ to $d u$. Thus the eigenvalue problem (1.1) on $\mathcal{H}$ is equivalent to (2.2) on the Hilbert space $L^{2}((0, \pi), d u)$. The boundary conditions (1.3) at $u=0$ (and similarly at $u=\pi$ ) can be written as

$$
\left\{\begin{aligned}
\lim _{u \searrow 0}\left(u^{-\frac{1}{2}} Y\right)^{\prime}(u) & =0 & & \text { if } k=0 \\
\lim _{u \searrow 0} u^{-\frac{1}{2}} Y(u) & =0 & & \text { if } k \neq 0 .
\end{aligned}\right.
$$

The Sturm-Liouville equation (2.2) is singular at the two end points $u=0, \pi$. An asymptotic expansion near $u=0$ shows that (2.2) has fundamental solutions $Y_{1 / 2}$ of the following form,

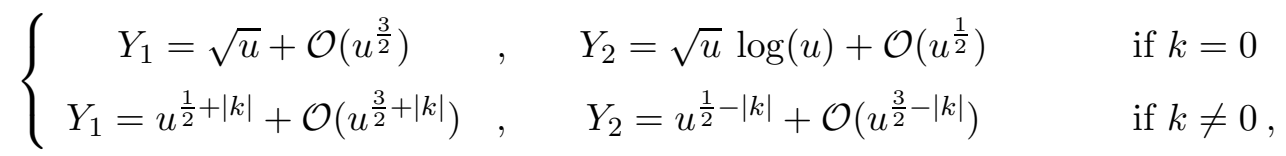

and similary at $u=\pi$. In the case $k \neq 0, Y_{1}$ is square integrable near $u=0$, whereas $Y_{2}$ is not. Thus, using Weyl's notation, the Sturm-Liouville operator is in the limit point case at both end points, and thus $\mathcal{A}$ is essentially selfadjoint (see [2, Sections 9.2, 9.3] or [3, Chapter XIII.2]). In the case $k=0$, on the other hand, both fundamental solutions are square integrable. This is the limit circle case, and the von-Neumann boundary conditions (2.5) choose a unique self-adjoint extension (see [2, Sections 9.4] or [3, Chapter XIII.2]). We conclude that the Sturm-Liouville operator in (2.2) has a unique self-adjoint extension in $L^{2}((0, \pi))$ which satisfies the boundary conditions (2.5). Hence the spectral theorem for unbounded operators in Hilbert spaces gives us the desired spectral representation of $\mathcal{A}$.

For each $\lambda \in \mathbb{R}$, there are (up to a constant) unique solutions of the ODE which satisfy the boundary conditions at $u=0$ and $u=\pi$, respectively. If the Wronskian of these two solutions vanishes, we obtain an eigenfunction in $L^{2}((0, \pi))$. Otherwise, these two solutions can be used to define the resolvent (see [3, XIII.3]), which is compact (see [3, XIII.4]). This shows that the operator $\mathcal{A}$ has a purely discrete spectrum consisting of simple eigenvalues without limit points. The positivity of $A$ is obvious from (1.2).

Finally, the boundary value problem 2.2. 2.5) is invariant under the transformation $u \rightarrow \pi-u$. Hence the parity subspaces $L^{ \pm}:=\left\{\phi \in L^{2}((0, \pi)) \mid \phi(\pi-u)= \pm \phi(u)\right\}$ are invariant under $\mathcal{A}$. This concludes the proof of Theorem 1.2

Clearly, the eigenfunctions $Y^{ \pm}$of even and odd parity satisfy at $u=\frac{\pi}{2}$ the boundary conditions

$$
\left\{\begin{array}{l}
\left(Y_{n}^{+}\right)^{\prime}\left(\frac{\pi}{2}\right)=0 \\
Y_{n}^{-}\left(\frac{\pi}{2}\right)=0
\end{array}\right.
$$

This makes it possible to consider instead of the the interval $(0, \pi)$ only the interval $\left(0, \frac{\pi}{2}\right]$ together with the boundary conditions (2.5, 2.7). In what follows, we shall always consider the boundary value problem (2.2, 2.5, 2.7).

In order to better understand Theorem [1.3, it is useful to consider the limits $n \rightarrow \infty$ and $\Omega \rightarrow \infty$. For fixed $\Omega$ and large $n$, Weyl's asymptotics applies and yields that the 
eigenvalues of $\mathcal{A}$ behave for large $n$ like the eigenvalues of the operator $-\frac{d^{2}}{d u^{2}}$,

$$
\lambda_{n}^{ \pm} \sim n^{2} \quad \text { and } \quad \lambda_{n+1}^{ \pm}-\lambda_{n}^{ \pm} \sim n
$$

Therefore, it is obvious that the statement of Theorem 1.3 holds for any fixed $\Omega$ and sufficiently large $N=N(\Omega)$. The estimate

$$
\left|\lambda_{n}(\Omega)-\lambda_{n}\left(\Omega^{\prime}\right)\right| \leq\left\|\mathcal{A}(\Omega)-\mathcal{A}\left(\Omega^{\prime}\right)\right\|_{\infty} \leq\left|\Omega-\Omega^{\prime}\right|\left(\Omega+\Omega^{\prime}+2|k|\right)
$$

yields that eigenvalues of $\mathcal{A}$ are locally Lipschitz in $\Omega$, uniformly in $n$. This shows that the constant $N(\Omega)$ can be chosen locally uniformly in $\Omega$. If conversely we fix $n$, the $n$th spheroidal eigenvalue $\lambda_{n}$ has for large $\Omega$ the asymptotic expansion (see [5] or [1] )

$$
\lambda_{n}(\Omega)=\left\{\begin{aligned}
2(n+1) \Omega+O(1) & \text { if } n-k \text { is even }, \\
2 n \Omega+O(1) & \text { if } n-k \text { is odd } .
\end{aligned}\right.
$$

Hence for each $n$, we can make the eigenvalue gap arbitrarily large by choosing $\Omega$ sufficiently large. We conclude that it remains to show that the eigenvalue gaps are bounded uniformly as both $N$ and $|\Omega|$ become large. This is the hard part of Theorem 1.3. and we state it as a separate Lemma.

Lemma 2.1 For any given $k \in \mathbb{Z}$ and $c>0$, there are constants $N \in \mathbb{N}$ and $\Omega_{0}>0$ such that

$$
\lambda_{n+1}^{ \pm}-\lambda_{n}^{ \pm}>c \quad \text { for all } n \geq N \text { and } \Omega>\Omega_{0} .
$$

The proof of this lemma requires detailed eigenvalue estimates. We will complete it in Section 7 , and this will also finish the proof of Theorem 1.3.

Finally, the node theorem [15, Theorem 14.10] tells us about the number of zeros of the spheroidal wave functions. In our setting, the statement of the node theorem can easily be derived as follows. Using the initial conditions (2.5) together with (2.8), we obtain from the Picard-Lindelöf theorem that the eigenfunctions $Y_{n}^{ \pm}$corresponding to the eigenvalue $\lambda_{n}^{ \pm}$depend smoothly on the parameter $\Omega$. Furthermore, the asymptotics near the boundaries (2.5] 2.7) shows that the functions $Y_{n}^{ \pm}$have no zeros in the intervals $(0, \varepsilon)$ and $\left(\frac{\pi}{2}-\varepsilon, \frac{\pi}{2}\right)$, for a parameter $\varepsilon>0$ which depends continuously on $\Omega$. Thus, if the number of zeros of the function $Y_{n}^{ \pm}$changed at some $\Omega$, there would be a $u \in(0, \pi)$ with $Y_{n}^{ \pm}(u)=0=\left(Y_{n}^{ \pm}\right)^{\prime}(u)$, in contradiction to the fact that $Y_{n}^{ \pm}$does not vanish identically. We conclude that the number of zeros of $Y_{n}^{ \pm}$on $\left(0, \frac{\pi}{2}\right)$ is independent of $\Omega$, and therefore it suffices to consider the case $\Omega=0$, when the spheroidal wave functions reduce to the Legendre polynomials. Counting their zeros, we conclude that the function

$$
Y_{n}^{ \pm} \text {has } n \text { zeros on }\left(0, \frac{\pi}{2}\right) \text {. }
$$

\section{The Complex Riccati Equation}

Let $Y_{1}$ and $Y_{2}$ be two real fundamental solutions of the Sturm-Liouville equation (2.2) for a general real and smooth potential $V$. Then their Wronskian

$$
w:=Y_{1}(u) Y_{2}^{\prime}(u)-Y_{1}^{\prime}(u) Y_{2}(u)
$$

is a constant; we assume in what follows that $w>0$. We combine the two real solutions to the complex function

$$
z=Y_{1}+i Y_{2},
$$


and denote its polar decomposition by

$$
z=\rho e^{i \varphi}
$$

with real functions $\rho(u) \geq 0$ and $\varphi(u)$. By linearity, $z$ is a complex solution of the SturmLiouville equation

$$
z^{\prime \prime}=V z .
$$

Note that $z$ has no zeros because at every $u$ at least one of the fundamental solutions does not vanish. Thus the function $y$ defined by

$$
y=\frac{z^{\prime}}{z}
$$

is smooth. Moreover, $y$ satisfies the complex Riccati equation

$$
y^{\prime}+y^{2}=V .
$$

The fact that the solutions of the complex Riccati equation are smooth will be helpful for getting estimates. Conversely, from a solution of the Riccati equation one obtains the corresponding solution of the Sturm-Liouville equation by integration,

$$
\left.\log z\right|_{u} ^{v}=\int_{u}^{v} y
$$

Using (3.1) in (3.3) gives separate equations for the amplitude and phase of $z$,

$$
\rho^{\prime}=\rho \operatorname{Re} y, \quad \varphi^{\prime}=\operatorname{Im} y,
$$

and integration gives

$$
\begin{aligned}
\left.\log \rho\right|_{u} ^{v} & =\int_{u}^{v} \operatorname{Re} y \\
\left.\varphi\right|_{u} ^{v} & =\int_{u}^{v} \operatorname{Im} y .
\end{aligned}
$$

Furthermore, the Wronskian yields a simple algebraic relation between $\rho$ and $y$. Namely, $w$ can be expressed by $w=\operatorname{Im}\left(\bar{z} z^{\prime}\right)=\rho^{2} \operatorname{Im} y$ and thus

$$
\rho^{2}=\frac{w}{\operatorname{Im} y} .
$$

Since $\rho^{2}$ and $w$ are non-negative, we see that

$$
\operatorname{Im} y(u)>0 \quad \text { for all } u \text {. }
$$

The boundary conditions for the Sturm-Liouville equation can easily be translated into conditions for $y$. To this end we write the solutions $Y_{n}^{ \pm}$of the Sturm-Liouville equation corresponding to the eigenvalues $\lambda_{n}^{ \pm}$as $Y_{n}^{ \pm}=\operatorname{Im}\left(e^{-i \alpha} z_{n}^{ \pm}\right)$with a suitable phase factor $e^{-i \alpha}$. Then a Dirichlet condition can be written as $\varphi=\alpha \bmod \pi$. A Neumann boundary condition gives

$$
\begin{aligned}
0 & =\operatorname{Im}\left(e^{-i \alpha} y z\right)=\operatorname{Re}\left(e^{-i\left(\alpha+\frac{\pi}{2}\right)} y z\right) \\
& =\left[\operatorname{Re} y \cos \left(\varphi-\alpha-\frac{\pi}{2}\right)-\operatorname{Im} y \sin \left(\varphi-\alpha-\frac{\pi}{2}\right)\right] \rho
\end{aligned}
$$


and thus

$$
\varphi=\alpha+\frac{\pi}{2}+\arctan \left(\frac{\operatorname{Re} y}{\operatorname{Im} y}\right)
$$

According to (3.7) and (3.9) the function $\varphi(u)$ is monotone increasing. Therefore, the number of zeros of $Y$, 2.10) tells us how often $\varphi$ crossed the points $\bmod \pi$. This allows us to completely determine the "phase shifts" on the interval $\left(0, \frac{\pi}{2}\right)$,

$$
\begin{aligned}
& \left.\varphi_{n}^{+}\right|_{0} ^{\frac{\pi}{2}}=\frac{\pi}{2}+\arctan \left(\frac{\operatorname{Re} y\left(\frac{\pi}{2}\right)}{\operatorname{Im} y\left(\frac{\pi}{2}\right)}\right)+n \pi \\
& \left.\varphi_{n}^{-}\right|_{0} ^{\frac{\pi}{2}}=(n+1) \pi
\end{aligned}
$$

(we use the usual convention that the arc tangent takes values in $\left(-\frac{\pi}{2}, \frac{\pi}{2}\right)$ ). Using (3.7) these boundary conditions can be expressed purely in terms of $y$ and the integral of the imaginary part of $y$.

For the gap estimates we need to control how $y$ depends on $\lambda$. To this end, we differentiate through the complex Riccati equation (3.4) and use that $\partial_{\lambda} V=-1$ according to (2.3) and (2.4). This gives the linear ODE

$$
y_{\lambda}^{\prime}=-1-2 y y_{\lambda},
$$

where the $\lambda$-derivative is denoted by a subscript. This equation can immediately be integrated using variation of constants. Applying (3.5), we obtain

$$
\left.z^{2} y_{\lambda}\right|_{u} ^{v}=-\int_{u}^{v} z^{2}
$$

Substituting the integration-by-parts formula

$$
\int_{u}^{v} z^{2}=\int_{u}^{v} \frac{1}{2 y}\left(z^{2}\right)^{\prime}=\left.\frac{z^{2}}{2 y}\right|_{u} ^{v}+\int_{u}^{v} \frac{V-y^{2}}{2 y^{2}} z^{2}
$$

we obtain the identity

$$
\left.z^{2} y_{\lambda}\right|_{u} ^{v}=-\left.\frac{z^{2}}{2 y}\right|_{u} ^{v}-\int_{u}^{v} \frac{V-y^{2}}{2 y^{2}} z^{2} .
$$

In our estimates we will work both with (3.12) and (3.13).

\section{Invariant Disk Estimates}

In this section we describe estimates for the complex Riccati equation (3.4) with initial conditions at $u=0$,

$$
y^{\prime}=V-y^{2}, \quad y(0)=y_{0}
$$

on the interval $\left[0, u_{\max }\right)$ with $u_{\max } \in \mathbb{R}^{+} \cup\{\infty\}$. In what follows, the potential $V \in$ $C^{\infty}\left(\left[0, u_{\max }\right)\right)$ can be any real and smooth function. The next lemma is the key to all the estimates in this section. 
Lemma 4.1 Let $\alpha$ be a real function on $\left[0, u_{\max }\right)$ which is continuous and piecewise $C^{1}$. For a constant $T_{0} \geq 1$ we introduce the functions $\sigma, U$ and $T$ by

$$
\begin{aligned}
\sigma(u) & =\exp \left(2 \int_{0}^{u} \alpha\right) \\
U(u) & =V-\alpha^{2}-\alpha^{\prime} \\
T(u) & =T_{0} \exp \left(\frac{1}{2} T V_{[0, u)} \log \left|\sigma^{2} U\right|\right) .
\end{aligned}
$$

Furthermore, we define the functions $\beta, R$ and $m$ by

$$
\begin{aligned}
\beta & =\frac{\sqrt{|U|}}{2}\left(T+\frac{1}{T}\right) \\
R & =\frac{\sqrt{|U|}}{2}\left(T-\frac{1}{T}\right) \\
m & =\alpha+i \beta .
\end{aligned}
$$

Suppose that $U \leq 0$ on $\left[0, u_{\max }\right)$. If a solution $y$ of the boundary value problem (4.1) satisfies at $u=0$ the condition

$$
|y-m| \leq R
$$

then this condition holds for all $u \in\left[0, u_{\max }\right)$.

Before coming to the proof, we briefly discuss the statement of this lemma. If $\alpha$ is a real solution of the Riccati equation, the function $U$ as given by (4.3) vanishes identically, and thus $\beta \equiv 0 \equiv R$. In this case, the above lemma reduces to the trivial statement that $y(0)=\alpha$ implies that $y=\alpha$ on $\left[0, u_{\max }\right)$. It is more interesting to consider the case that $\alpha=\operatorname{Re} y$ with $y$ a complex solution of the Riccati equation. Then

$$
U=\operatorname{Re}\left(V-\alpha^{2}-\alpha^{\prime}\right)=\operatorname{Re}\left(V-y^{2}-y^{\prime}\right)-(\operatorname{Im} y)^{2}=-(\operatorname{Im} y)^{2}<0 .
$$

Moreover, from (3.6) we can immediately compute $\sigma$,

$$
\sigma(u)=\exp \left(2 \int_{0}^{u} \operatorname{Re} y\right)=\frac{\rho^{2}(u)}{\rho^{2}(0)} .
$$

Substituting these relations into (3.8) yields

$$
\sigma^{2} U=-\frac{\rho^{4}(u)}{\rho^{4}(0)}(\operatorname{Im} y)^{2}=-\frac{w^{2}}{\rho^{4}(0)} .
$$

Hence the function $\log \left|\rho^{2} U\right|$ is a constant, and its total variation in (4.4) vanishes. This means that $T$ is a constant, and thus $\beta$ and $R$ are constant multiples of $\operatorname{Im} y$. Our Lemma states that the circles of radius $R(u)$ around the point $m(u)=\alpha(u)+i \beta(u)$ are invariant under the flow of the Riccati equation.

If no solution of the Riccati equation is known (and this will of course be the usual situation), one can take for $\alpha$ the real part of an approximate solution of the complex Riccati equation. In this case, the function $\log \left|\rho^{2} U\right|$ will not be constant, but we can hope that its total variation is small. If this is the case, our lemma gives an "improved approximative solution" $m$ together with a rigorous error estimate $R$. A good candidate for an approximate solution would be the usual wave function obtained by "gluing together" 
suitable WKB wave functions and Airy functions as used in the semi-classical analysis of one-dimensional Schrödinger problems. We remark that the above lemma might even be useful for getting rigorous error estimates for numerical solutions of the Sturm-Liouville or Riccati equations. In this case, one would have to estimate the total variation of $\log \left|\rho^{2} U\right|$ from above, and this might be doable numerically if one has some control of the accuracy of the numerical calculation.

Proof of Lemma 4.1. For $\varepsilon>0$ we set

$$
T_{\varepsilon}(u)=T_{0} \exp \left(\frac{1}{2} \int_{0}^{u}\left|\frac{\left|\sigma^{2} U\right|^{\prime}}{\left|\sigma^{2} U\right|}\right|+\varepsilon e^{-u}\right)
$$

and let $R_{\varepsilon}$ and $m_{\varepsilon}$ be the functions obtained from (4.5)-4.7) if one replaces $T$ by $T_{\varepsilon}$. Since $T_{\varepsilon}(0)=T(0)$ and $\lim _{\varepsilon \backslash 0} T_{\varepsilon}=T$, it suffices to show that for all $\varepsilon>0$ the following statement holds,

$$
\left|y-m_{\varepsilon}\right|(0) \leq R_{\varepsilon}(0) \quad \Longrightarrow \quad\left|y-m_{\varepsilon}\right|(u) \leq R_{\varepsilon}(u) \text { for all } u \in\left[0, u_{\max }\right) .
$$

In order to prove this statement, we will show that the assumption

$$
\left|y-m_{\varepsilon}\right|(u)=R_{\varepsilon}(u)
$$

implies that

$$
\left|y-m_{\varepsilon}\right|^{\prime}(u)<R_{\varepsilon}^{\prime}(u) .
$$

In what follows we will often omit the subscript $\varepsilon$.

Assume that (4.10) holds and that $U \leq 0$. Then we can represent $y$ as

$$
y=m+R e^{i \varphi}
$$

with $\varphi \in[0,2 \pi)$. Furthermore, it follows immediately from (4.5), (4.6), and (4.3) that

$$
R^{2}=U+\beta^{2}=V-\alpha^{2}+\beta^{2}-\alpha^{\prime} .
$$

Using the above relations together with (3.4), we obtain

$$
\begin{aligned}
& \frac{1}{2} \frac{d}{d u}|y-m|^{2}=(\operatorname{Re} y-\alpha)(\operatorname{Re} y-\alpha)^{\prime}+(\operatorname{Im} y-\beta)(\operatorname{Im} y-\beta)^{\prime} \\
& \stackrel{\text { 3.4 }}{=} \quad(\operatorname{Re} y-\alpha)\left[V-(\operatorname{Re} y)^{2}+(\operatorname{Im} y)^{2}-\alpha^{\prime}\right]-(\operatorname{Im} y-\beta)\left[2 \operatorname{Re} y \operatorname{Im} y+\beta^{\prime}\right] \\
&=\quad(\operatorname{Re} y-\alpha)\left[V-(\operatorname{Re} y)^{2}-(\operatorname{Im} y)^{2}+2 \beta \operatorname{Im} y-\alpha^{\prime}\right] \\
& \quad+(\operatorname{Re} y-\alpha) 2(\operatorname{Im} y-\beta) \operatorname{Im} y \\
& \quad-(\operatorname{Im} y-\beta)\left[\beta^{\prime}+2 \alpha \operatorname{Im} y\right]-(\operatorname{Im} y-\beta) 2(\operatorname{Re} y-\alpha) \operatorname{Im} y \\
&=\quad(\operatorname{Re} y-\alpha)\left[V-(\operatorname{Re} y-\alpha)^{2}-(\operatorname{Im} y-\beta)^{2}-\alpha^{2}+\beta^{2}-\alpha^{\prime}\right] \\
&-(\operatorname{Im} y-\beta)\left[\beta^{\prime}+2 \alpha \beta\right]-2 \alpha\left((\operatorname{Re} y-\alpha)^{2}+(\operatorname{Im} y-\beta)^{2}\right) \\
& \quad R \cos \varphi\left[V-R^{2}+|m|^{2}-\alpha^{\prime}-2 \alpha^{2}\right]-R \sin \varphi\left[\beta^{\prime}+2 \alpha \beta\right]-2 \alpha R^{2} \\
& \quad-2 \alpha R^{2}-R\left(\beta^{\prime}+2 \alpha \beta\right) \sin \varphi \leq-2 \alpha R^{2}+R\left|\beta^{\prime}+2 \alpha \beta\right| .
\end{aligned}
$$

Using that $\frac{d}{d u}|y-m|^{2}=2 R|y-m|^{\prime}$, we obtain the simple inequality

$$
|y-m|^{\prime} \leq-2 \alpha R+\left|\beta^{\prime}+2 \alpha \beta\right| .
$$


Hence in order to prove (4.11), it suffices to show that

$$
R^{\prime}>-2 \alpha R+\left|\beta^{\prime}+2 \alpha \beta\right| .
$$

Using (4.2), we write the last inequality in the equivalent form

$$
(\sigma R)^{\prime}>\left|(\sigma \beta)^{\prime}\right|
$$

In order to prove this inequality, we first use (4.5) and (4.6) to write the functions $\sigma \beta$ and $\sigma R$ as

$$
\begin{aligned}
\sigma \beta & =\frac{1}{2}\left(\sqrt{\left|\sigma^{2} U\right|} T+\sqrt{\left|\sigma^{2} U\right|} T^{-1}\right) \\
\sigma R & =\frac{1}{2}\left(\sqrt{\left|\sigma^{2} U\right|} T-\sqrt{\left|\sigma^{2} U\right|} T^{-1}\right) .
\end{aligned}
$$

By definition of $T_{\varepsilon}$ (4.9),

$$
\frac{T^{\prime}}{T}=\frac{1}{2}\left|\frac{\left|\sigma^{2} U\right|^{\prime}}{\left|\sigma^{2} U\right|}\right|+\varepsilon e^{-u} .
$$

It follows that

$$
\left\{\begin{aligned}
\left(\sqrt{\left|\sigma^{2} U\right|} T^{-1}\right)^{\prime} & =-\varepsilon e^{-u}\left(\sqrt{\left|\sigma^{2} U\right|} T^{-1}\right) & & \text { if }\left|\sigma^{2} U\right|^{\prime} \geq 0 \\
\left(\sqrt{\left|\sigma^{2} U\right|} T\right)^{\prime} & =\varepsilon e^{-u}\left(\sqrt{\left|\sigma^{2} U\right|} T\right) & & \text { if }\left|\sigma^{2} U\right|^{\prime}<0 .
\end{aligned}\right.
$$

Hence when we differentiate through (4.15) and set $\varepsilon=0$, either the first or the second summand drop out in each equation, and we obtain $(\sigma R)^{\prime}=|\sigma \beta|^{\prime}$. If $\varepsilon>0$, an inspection of the signs of the additional terms gives (4.14).

The question arises how the function $\alpha$ in the above lemma is to be chosen. At this point, it is very helpful to regard (2.2) as the one-dimensional Schrödinger equation for a quantum mechanical wave function $Y$, because this makes it possible to use ideas from semi-classical analysis. In order to explain our method, we first consider the WKB wave functions 6

$$
\phi(u)=|V|^{-\frac{1}{4}} \exp \left( \pm i \int^{u} \sqrt{|V|}\right),
$$

which should be good approximations to fundamental solutions in the "semiclassical regime" $V \ll 0$. The corresponding function $y$ is

$$
y(u)=\frac{\phi^{\prime}}{\phi}= \pm i \sqrt{|V|}-\frac{V^{\prime}}{4 V} .
$$

Lemma 4.1 should give a good estimate only if $m$ is close to the exact solution $y$. This leads us to choose for the function $\alpha=\operatorname{Re} m$ the corresponding expression in the WKB approximation,

$$
\alpha=-\frac{V^{\prime}}{4 V} \quad \text { in the "semiclassical regime". }
$$

This gives rise to the following estimate. 
Theorem 4.2 Assume that the potential $V$ is negative and monotone increasing on the interval $\left[0, u_{\max }\right)$, and that the following condition holds,

$$
K:=\frac{\sup \left|V^{\prime \prime}\right|+\mathrm{TV} V^{\prime \prime}}{V_{\max }^{2}}+\sup \frac{V^{\prime 2}}{|V|^{3}} \leq 1,
$$

where $V_{\max }:=\sup V \leq 0$ (and the supremum as well as the total variation are taken on the interval $\left[0, u_{\max }\right)$ ). Then the solution $y$ of the boundary value problem (4.1) with initial condition

$$
y_{0}=i \sqrt{|V(0)|}-\frac{V^{\prime}(0)}{4 V(0)}
$$

satisfies on $\left[0, u_{\max }\right)$ the inequalities

$$
\begin{aligned}
\left|y-i \sqrt{|V|}+\frac{V^{\prime}}{4 V}\right| & \leq 20 \sqrt{|V|} K \\
\operatorname{Im} y & \geq \frac{\sqrt{|V|}}{10} .
\end{aligned}
$$

Proof. We introduce on $\left[0, u_{\max }\right]$ the function $\alpha$ by

$$
\alpha(u)=-\frac{V^{\prime}}{4 V} .
$$

Then from (4.3),

$$
\begin{aligned}
\alpha^{\prime} & =-\frac{V^{\prime \prime}}{4 V}+\frac{V^{\prime 2}}{4 V^{2}} \\
U & =V\left(1+\frac{V^{\prime \prime}}{4 V^{2}}+\frac{5 V^{\prime 2}}{16|V|^{3}}\right) .
\end{aligned}
$$

Using the inequality (4.16) we get

$$
2 V \leq U \leq \frac{V}{2}
$$

In particular, $U$ is negative.

The inequalities (4.21) and (4.16) allow us to estimate $\sqrt{|U|}-\sqrt{|V|}$,

$$
|\sqrt{|U|}-\sqrt{|V|}|=\frac{|U-V|}{\sqrt{|U|}+\sqrt{|V|}} \leq \sqrt{|V|}\left|\frac{U-V}{V}\right| \leq \frac{\sqrt{|V|}}{2} K .
$$

Dividing by $\sqrt{|V|}$ and $\sqrt{|U|}$, we obtain furthermore

$$
\frac{1}{1+K} \leq \sqrt{\frac{|V|}{|U|}} \leq 1+K
$$

Choosing $T_{0}=1+K$, we have the following estimates at $u=0$,

$$
\begin{aligned}
|y-m| & =|\sqrt{|V|}-\beta|=\left|\sqrt{|V|}-\frac{\sqrt{|U|}}{2}\left((1+K)+\frac{1}{1+K}\right)\right| \\
& =\frac{\sqrt{|U|}}{2}\left|(1+K)+\frac{1}{1+K}-2 \sqrt{\frac{|V|}{|U|}}\right| .
\end{aligned}
$$


Applying (4.24) we obtain

$$
|y-m| \leq \frac{\sqrt{|U|}}{2}\left((1+K)-\frac{1}{1+K}\right)=R .
$$

We conclude that the inequality (4.8) holds at $u=0$.

Hence we can apply Lemma 4.1 and obtain that (4.8) holds for all $u \in\left[0, u_{\max }\right)$. Combining this with the inequalities (4.22) and (4.24) we obtain

$$
\begin{aligned}
\left|y-i \sqrt{|V|}+\frac{V^{\prime}}{4 V}\right| \leq|y-m|+|\beta-\sqrt{|V| \mid} \leq R+| \beta-\sqrt{|V| \mid} & \leq R+\frac{\sqrt{|U|}}{2}\left(T+\frac{1}{T}-2 \sqrt{\frac{|V|}{|U|}}\right)=\sqrt{|U|}\left(T-\sqrt{\frac{|V|}{|U|}}\right) \\
& =2 \sqrt{|V|}\left(T-\frac{1}{1+K}\right) \leq 2 \sqrt{|V|}(T-1+K) \\
& \leq \beta-R=\frac{\sqrt{|U|}}{T} \geq \frac{\sqrt{|V|}}{\sqrt{2} T} .
\end{aligned}
$$

It remains to estimate the function $T$, (4.4). We first compute $\sigma$ and $\sigma^{2} U$,

$$
\begin{aligned}
\sigma & =\sqrt{\frac{V_{0}}{V(u)}} \\
\frac{\sigma^{2} U}{\left|V_{0}\right|} & =-1-\frac{V^{\prime \prime}}{4 V^{2}}-\frac{5 V^{\prime 2}}{16|V|^{3}},
\end{aligned}
$$

where we set $V_{0}=V(0)$. Applying (4.16) we immediately obtain the inequalities

$$
\frac{1}{2} \leq\left|\frac{\sigma^{2} U}{V_{0}}\right| \leq 2
$$

The lower bound allows us to leave out the logarithm in the total variation in the definition of $T$; namely,

$$
\operatorname{TV}_{[0, u)} \log \left|\sigma^{2} U\right|=\int_{0}^{u}\left|\frac{\left(\sigma^{2} U\right)^{\prime}}{\sigma^{2} U}\right| \leq 2 \int_{0}^{u}\left|\frac{\left(\sigma^{2} U\right)^{\prime}}{\left|V_{0}\right|}\right|=2 \mathrm{TV}_{[0, u)} \frac{\sigma^{2} U}{\left|V_{0}\right|}
$$

We substitute (4.28) into this equation and estimate the total variation of the individual terms using (4.16) as well as the monotonicity of $V$,

$$
\begin{aligned}
\operatorname{TV}_{[0, u)} \frac{V^{\prime \prime}}{V^{2}} & \leq \int_{0}^{u} \frac{\left|V^{\prime \prime \prime}\right|}{V^{2}}+2 \int_{0}^{u} \frac{\left|V^{\prime \prime}\right| V^{\prime}}{(-V)^{3}} \leq \frac{\mathrm{TV} V^{\prime \prime}+\sup \left|V^{\prime \prime}\right|}{V_{\max }^{2}} \\
\operatorname{TV}_{[0, u)} \frac{V^{\prime 2}}{(|V|)^{3}} & \leq \int_{0}^{u} \frac{2\left|V^{\prime \prime}\right| V^{\prime}}{(|V|)^{3}}+\int_{0}^{u} \frac{3\left|V^{\prime}\right|^{3}}{V^{4}} \leq \frac{\sup \left|V^{\prime \prime}\right|}{V_{\max }^{2}}+\int_{0}^{u} \frac{3\left|V^{\prime}\right|^{3}}{V^{4}} .
\end{aligned}
$$

In the last term we can integrate by parts,

$$
\int_{0}^{u} \frac{3\left|V^{\prime}\right|^{3}}{V^{4}}=\int_{0}^{u} V^{\prime 2}\left((-V)^{-3}\right)^{\prime}=\left.\frac{V^{\prime 2}}{|V|^{3}}\right|_{0} ^{u}-\int_{0}^{u} \frac{2 V^{\prime \prime} V^{\prime}}{|V|^{3}} \leq \sup \frac{V^{\prime 2}}{|V|^{3}}+\frac{\sup \left|V^{\prime \prime}\right|}{V_{\max }^{2}} .
$$


Collecting all the terms and using (4.16) we conclude that

$$
\mathrm{TV}_{[0, u)} \log \left|\sigma^{2} U\right| \leq 2 K .
$$

We substitute this bound into (4.4) and use that $T_{0}=1+K$ to obtain the bound

$$
T-1=(1+K) e^{K}-1 \leq\left|e^{K}-1\right|+K e^{K} \leq 2 e K .
$$

Using this bound in (4.25) and (4.26) concludes the proof.

The condition (4.16) will clearly be violated when $|V|$ becomes small. This is not astonishing because the WKB approximation fails near the zeros of the potential. In this "quantum regime", there is no canonical candidate for $\alpha$, and therefore we simply take

$$
\alpha=\text { const in the "quantum regime". }
$$

We state the corresponding estimate in such a way that it can easily be "pasted together" with the result of Lemma 4.2 ,

Theorem 4.3 Assume that the potential $V$ is negative and monotone (increasing or decreasing) on $\left[0, u_{\max }\right)$, and that for some constant $\kappa>0$ the following condition holds,

$$
\sqrt{\left|V_{0}\right|} u_{\max } \leq \kappa
$$

(with $\left.V_{0}=V(0)\right)$. Then any solution $y$ of the boundary value problem 4.1) which is bounded by

$$
\left|y_{0}\right| \leq c_{1} \sqrt{\left|V_{0}\right|}, \quad \operatorname{Im} y_{0} \geq \frac{\sqrt{\left|V_{0}\right|}}{c_{1}}
$$

for a suitable constant $c_{1} \geq 1$ satisfies on $\left[0, u_{\max }\right)$ the inequalities

$$
|y| \leq c_{2} \sqrt{\|V\|_{\infty}}, \quad \operatorname{Im} y \geq \frac{1}{c_{2}} \frac{\left|V_{0}\right|}{\sqrt{\|V\|_{\infty}}}
$$

where $\|V\|_{\infty}:=\sup _{\left[0, u_{\max }\right)}|V|$ and $c_{2}$ is a constant which depends only on $\kappa$ and $c_{1}$.

Proof. Let $\alpha$ be the constant function $\alpha=\sqrt{\left|V_{0}\right|}$. Then the function $U=V-\alpha^{2}$ is clearly negative. A simple calculation shows that by choosing $T_{0}=2 c_{1}\left(1+c_{1}\right)^{2}$, we can arrange that $\left|y_{0}-m(0)\right| \leq R(0)$. Lemma 4.1 yields that $|y-m| \leq R$ for all $u \in\left[0, u_{\max }\right)$.

Since $\alpha$ is a constant, the function $\sigma$ is given by $\sigma(u)=e^{2 \alpha u}$ and thus

$$
\left|\sigma^{2} U\right|=e^{4 \alpha u}\left(\alpha^{2}-V\right)
$$

As a consequence,

$$
\frac{\left|\sigma^{2} U\right|^{\prime}}{\left|\sigma^{2} U\right|} \leq 4 \alpha-\frac{V^{\prime}}{\alpha^{2}-V}
$$

If we integrate and use (4.29), we obtain the following bound for $T$,

$$
\begin{aligned}
T & \leq T_{0} e^{2 \alpha u}\left(\frac{\sqrt{\alpha^{2}-V_{0}}}{\sqrt{\alpha^{2}-V}}+\frac{\sqrt{\alpha^{2}-V}}{\sqrt{\alpha^{2}-V_{0}}}\right) \\
& \leq T_{0} e^{2 \alpha u} \frac{4 \sqrt{\|V\|_{\infty}}}{\alpha} \leq 4 T_{0} e^{2 \kappa} \sqrt{\frac{\|V\|_{\infty}}{\left|V_{0}\right|}}=: T_{\max } .
\end{aligned}
$$


Finally, we bound $y$ by

$$
\begin{aligned}
|y| & \leq|y-m|+|m| \leq R+\alpha+\beta \\
& =\sqrt{|U|} T+\alpha \leq(2 T+1) \alpha \\
\operatorname{Im} y & \geq \beta-R=\frac{\sqrt{|U|}}{T} \geq \frac{\alpha}{T} .
\end{aligned}
$$

These are the desired inequalities if we set $c_{2}=2 T_{\max }+1=8 c_{1}\left(1+c_{1}\right)^{2} e^{2 \kappa}+1$.

It is obvious from (2.3, 2.4 that the potential $V$ has a singularity at $u=0$. We now explain how Lemma 4.1 can be used for estimates near such a singular point. We will restrict attention to the case $k=0$, but our method applies similarly to general $k$. In order to find a good candidate for the function $\alpha$, we consider on the interval $\left[0, u_{\max }\right)$ the Sturm-Liouville equation with a potential which at $u=u_{\max }$ has the same singular behavior as (2.3),

$$
z^{\prime \prime}(u)=-\frac{1}{4\left(u_{\max }-u\right)^{2}} z
$$

Setting $v=u_{\max }-u$, this differential equation has the two fundamental solutions $\phi_{1}=\sqrt{v}$ and $\phi_{2}=\sqrt{v} \log v$, and therefore the function

$$
z=\sqrt{v}(1-i \log v)
$$

is a complex solution. The corresponding solution of the complex Riccati equation is given by

$$
y=\frac{z^{\prime}}{z}=-\frac{1}{2 v}+\frac{i}{v(1-i \log v)}=\left(-\frac{1}{2 v}-\frac{\log v}{v\left(1+\log ^{2} v\right)}\right)+\frac{i}{v\left(1+\log ^{2} v\right)} .
$$

Choosing $\alpha$ equal to the real part of this function gives rise to the following estimate.

Lemma 4.4 Suppose that the potential $V$ is on $\left[0, u_{\max }\right)$ of the form

$$
V=-\frac{1}{4} \frac{1}{\left(u_{\max }-u\right)^{2}}+B(u)
$$

with a function $B$ which is monotone (decreasing or increasing) and satisfies the inequality

$$
u_{\text {max }}^{2}\left(1+\log ^{2} u_{\text {max }}\right)^{2}\|B\|_{\infty} \leq \frac{1}{8}
$$

$\left(\right.$ with $\left.\|B\|_{\infty}:=\sup _{\left[0, u_{\max }\right)}|B|\right)$. Then any solution $y$ of the boundary value problem (4.1) with initial conditions bounded by

$$
\left|y_{0}\right| \leq C \sqrt{\left|V_{0}\right|}, \quad \operatorname{Im} y_{0} \geq \frac{\sqrt{\left|V_{0}\right|}}{C}
$$

for any constant $C \geq 1$ satisfies on $\left[0, u_{\max }\right)$ the inequalities

$$
\begin{aligned}
|y| & \leq \frac{64 C^{3}}{u_{\max }-u} \\
\operatorname{Im} y & \leq 64 C^{3}\left(1+\log ^{2} u_{\max }\right) \frac{1}{\left(u_{\max }-u\right) \log ^{2}\left(u_{\max }-u\right)} \\
\operatorname{Im} y & \geq \frac{1}{64 C^{3}\left(1+\log ^{2} u_{\max }\right)} \frac{1}{\left(u_{\max }-u\right) \log ^{2}\left(u_{\max }-u\right)} .
\end{aligned}
$$


Proof. We set $v=u_{\max }-u$ and choose for $\alpha$ the real function

$$
\alpha=-\frac{1}{2 v}-\frac{\log v}{v\left(1+\log ^{2} v\right)} .
$$

Using that $\alpha=\operatorname{Re} y$ with $y$ according to (4.31) and that $y$ is a solution of the complex Riccati equation corresponding to the Sturm-Liouville equation (4.30), we obtain

$$
U=V-\alpha^{2}-\alpha^{\prime}=\operatorname{Re}\left(V-y^{2}-y^{\prime}\right)-(\operatorname{Im} y)^{2}=B-\frac{1}{v^{2}\left(1+\log ^{2} v\right)^{2}} .
$$

Using the assumption (4.32) together with the fact that the function $v^{2}\left(1+\log ^{2} v\right)$ is monotone increasing, we obtain that $U$ is negative.

At $u=0$, the potentials $V$ and $U$ can easily be bounded from above and below,

$$
\begin{aligned}
& -\frac{3}{2} \leq-1-4 v^{2}|B| \leq l 4 v^{2} V=-1+4 v^{2}|B| \leq-\frac{1}{2} \\
& -\frac{3}{2} \leq 4 v^{2}\left(1+\log ^{2} v\right)^{2} U=-1+v^{2}\left(1+\log ^{2} v\right)^{2} B \leq-\frac{1}{2}
\end{aligned}
$$

and in particular

$$
\frac{1}{2} \leq \frac{\left(1+\log ^{2} v\right) \sqrt{|U|}}{|\sqrt{V}|} \leq 2 .
$$

A simple calculation shows that by choosing $T_{0}=2 C(1+C)^{2}\left(1+\log ^{2} u_{\max }\right)$, we can arrange that $\left|y_{0}-m(0)\right| \leq R(0)$. Lemma 4.1 yields that $|y-m| \leq R$ for all $u \in\left[0, u_{\max }\right)$.

Writing the function $\alpha$ in the form

$$
\alpha=\frac{d}{d u} \log \left(\sqrt{v\left(1+\log ^{2} v\right)}\right)
$$

we can immediately compute $\sigma^{2} U$,

$$
\begin{aligned}
\sigma^{2} & =v^{2}\left(1+\log ^{2} v\right)^{2} \\
\left|\sigma^{2} U\right| & =1-v^{2}\left(1+\log ^{2} v\right)^{2} B .
\end{aligned}
$$

Using the bound (4.32), we obtain

$$
\mathrm{TV}_{[0, u)} \log \left|\sigma^{2} U\right| \leq 2 \mathrm{TV}_{[0, u)}\left|\sigma^{2} U\right| \leq 4 u_{\max }^{2}\left(1+\log ^{2} u_{\max }\right)^{2}\|B\|_{\infty} \leq 2,
$$

and thus $T$ is bounded by $T \leq T_{0} e^{2} \leq 64 C^{3}\left(1+\log ^{2} u_{\max }\right)$. Finally, we combine the above estimates with the inequalities

$$
|y| \leq R+|\alpha|+\beta, \quad R-\beta \leq \operatorname{Im} y \leq R+\beta .
$$

The estimate (4.34) is very useful because it shows that the pole of $\operatorname{Im} y$ at $u=0$ is integrable. 


\section{Convexity Estimates}

The estimates of the previous section gave us good control of the solutions of the boundary value problem (4.1) provided that the potential is negative. In this section we proceed with estimates in the case that $V$ is positive, $V \geq 0$. Under this assumption, it is a simple observation that $\rho^{2}$ is convex, because

$$
\left(\rho^{2}\right)^{\prime \prime}=(\bar{z} z)^{\prime \prime}=2\left(V+|y|^{2}\right) \rho^{2}>0 .
$$

This fact will be essential for the estimates in this section.

We begin with a lemma which bounds $\rho$ from below.

Lemma 5.1 Suppose that $V$ is positive and monotone increasing on $\left[0, u_{\max }\right)$. Then every solution of the boundary value problem (4.1) satisfies on $\left[0, u_{\max }\right)$ the inequality

$$
\rho \geq \rho_{0} \frac{\operatorname{Im} y_{0}}{\left|y_{0}\right|}
$$

(with $\rho_{0}=|z(0)|$ and $z$ any solution of the corresponding complex Sturm-Liouville equation (3.2)).

Proof. Differentiating the equation $\rho^{\prime}=\rho \operatorname{Re}(y)$ and using the complex Riccati equation (3.4), we get

$$
\rho^{\prime \prime}=\rho(\operatorname{Re} y)^{2}+\rho \operatorname{Re}\left(V-y^{2}\right)=\left(V+(\operatorname{Im} y)^{2}\right) \rho,
$$

and using (3.8) we obtain the following differential equation for $\rho$,

$$
\rho^{\prime \prime}=V \rho+\frac{w^{2}}{\rho^{3}} .
$$

We let $\underline{\rho}(u)$ be the solution of the boundary value problem

$$
\underline{\rho}^{\prime \prime}=\frac{w^{2}}{\underline{\rho}^{3}} \quad \text { with } \quad \underline{\rho}(0)=\rho_{0}, \quad \underline{\rho}^{\prime}(0)=\rho^{\prime}(0) \equiv \rho_{0} \operatorname{Re} y_{0} .
$$

The function $\underline{\rho}$ is a solution of (5.2) in the case $V \equiv 0$. Therefore, $\underline{\rho}$ can be written explicitly in the form $\underline{\rho}=|\underline{z}|$ with $\underline{z}$ a solution of the complex Sturm-Liouville equation without potential with Wronskian equal to $w$, i.e.

$$
\underline{z}^{\prime \prime}=0 \quad \text { and } \quad \operatorname{Im}\left(\underline{\bar{z}} \underline{z}^{\prime}\right)=w=\rho_{0}^{2} \operatorname{Im} y_{0} .
$$

A short calculation shows that $\underline{\rho}$ has the simple form

$$
\underline{\rho}(u)=\rho_{0}\left|1+y_{0} u\right| .
$$

This function is defined even for all $u \in \mathbb{R}$. It is convex, and its minimum is computed to be

$$
\min _{u \in \mathbb{R}} \underline{\rho}(u)=\rho_{0} \frac{\operatorname{Im} y_{0}}{\left|y_{0}\right|} .
$$

We introduce the set $I \subset \mathbb{R}^{2}$ by

$$
I=\left\{\left(\underline{\rho}(x),\left[\underline{\rho}^{\prime}(x), \infty\right)\right) \text { with } x \in \mathbb{R}\right\} .
$$


Let us show that $I$ is an invariant region for $\rho$ in phase space, i.e. that for all $u \in\left[0, u_{\max }\right)$,

$$
\left(\rho(u), \rho^{\prime}(u)\right) \in I \text {. }
$$

Once this is shown, the lemma follows immediately from (5.4). Due to our initial condition, (5.5) is clearly satisfied at $u=0$. Thus assume that $[0, v]$ with $0 \leq v<u_{\max }$ is the maximal interval where (5.5) holds. Then the point $\left(\rho(v), \rho^{\prime}(v)\right)$ lies on the boundary of $I$. Using that $\underline{\rho}$ is convex and thus $\underline{\rho}$ is monotone increasing, one finds that either

$$
\rho(v)=\min \underline{\rho} \text { and } \rho^{\prime}(v)>0
$$

or else there is $x \in \mathbb{R}$ such that

$$
\rho(v)=\underline{\rho}(x) \text { and } \quad \rho^{\prime}(v)=\underline{\rho}^{\prime}(x) .
$$

In the first case, it is obvious that the gradient of $\left(\rho(v), \rho^{\prime}(v)\right)$ is pointed towards the interior of $I$. In the second case, the estimate

$$
\rho^{\prime \prime}(v)=V \rho(v)+\frac{w}{\rho(v)^{3}}>\frac{w}{\rho(v)^{3}}=\frac{w}{\underline{\rho}(x)^{3}}=\underline{\rho}^{\prime \prime}(x)
$$

yields that

$$
\rho^{\prime}(v)=\underline{\rho}^{\prime}(x) \quad \text { and } \quad \rho^{\prime \prime}(v)>\underline{\rho}^{\prime \prime}(x) .
$$

Hence the gradient of $\left(\rho(u), \rho^{\prime}(u)\right)$ again points towards the interior of $I$. We conclude that (5.5) holds also in an interval $[v, v+\varepsilon)$ with $\varepsilon>0$, a contradiction.

This lemma has the following immediate consequence. Due to the convexity of $\rho$,

$$
\sup _{[0, u)} \rho \leq \rho_{0}+\rho(u)=\rho(u)\left(1+\frac{\rho_{0}}{\rho(u)}\right) \leq \rho(u)\left(1+\frac{\left|y_{0}\right|}{\operatorname{Im} y_{0}}\right)
$$

and hence

$$
\sup _{[0, u)} \rho \leq \rho(u) \frac{2\left|y_{0}\right|}{\operatorname{Im} y_{0}} .
$$

In regions where the potential $V$ is large, we expect that $\rho$ should increase exponentially. The next lemma quantifies this exponential increase of $\rho$ by showing that in the "semiclassical regime" $V \gg 0$, the integral over $\rho^{2}$ is much smaller than the supremum of $\rho^{2}$.

Lemma 5.2 Suppose that $V$ is positive and monotone increasing on $\left[0, u_{\text {max }}\right)$. Then every solution of the boundary value problem (4.1) satisfies on $\left[0, u_{\max }\right)$ the inequality

$$
\int_{0}^{u} \rho^{2} \leq L \sup _{[0, u)} \rho^{2}
$$

with L given by

$$
L=\sup \left(\frac{3}{\sqrt{V}}+\frac{V^{\prime}}{V^{2}}\right)+\mathrm{TV} \frac{V^{\prime}}{V^{2}}
$$


Proof. We substitute the differential equation for $\rho^{2}$, (5.1), into the integral,

$$
\int_{0}^{u} \rho^{2}=\frac{1}{2} \int_{0}^{u} \frac{1}{V+|y|^{2}}\left(\rho^{2}\right)^{\prime \prime}
$$

Integrating by parts gives

$$
\int_{0}^{u} \rho^{2}=\left.\frac{\left(\rho^{2}\right)^{\prime}}{2\left(V+|y|^{2}\right)}\right|_{0} ^{u}-\frac{1}{2} \int_{0}^{u}\left(\frac{1}{V+|y|^{2}}\right)^{\prime}\left(\rho^{2}\right)^{\prime} .
$$

Using the estimates

$$
\begin{aligned}
\left|\frac{\left(\rho^{2}\right)^{\prime}}{V+|y|^{2}}\right| & \leq \frac{2 \rho^{2}|y|}{V+|y|^{2}} \leq \frac{2 \rho^{2}|y|}{2 \sqrt{V}|y|}=\frac{\rho^{2}}{\sqrt{V}} \\
\left|\left(\frac{1}{V+|y|^{2}}\right)^{\prime}\right| & \leq \frac{V^{\prime}+2|y|\left|V-y^{2}\right|}{\left(V+|y|^{2}\right)^{2}} \leq \frac{V^{\prime}}{V^{2}}+\frac{2|y|}{V+|y|^{2}} \leq \frac{V^{\prime}}{V^{2}}+\frac{1}{\sqrt{V}}
\end{aligned}
$$

we obtain

$$
\int_{0}^{u} \rho^{2} \leq \sup _{[0, u)} \frac{\rho^{2}}{\sqrt{V}}+\frac{1}{2} \int_{0}^{u}\left(\frac{V^{\prime}}{V^{2}}+\frac{1}{\sqrt{V}}\right)\left|\left(\rho^{2}\right)^{\prime}\right| .
$$

When integrating by parts once again we must be careful because the function $\left(\rho^{2}\right)^{\prime}$ may change signs. However, since $\rho^{2}$ is convex, it changes signs at most once, and therefore we get positive boundary terms at most twice,

$$
\int_{0}^{u}\left(\frac{V^{\prime}}{V^{2}}+\frac{1}{\sqrt{V}}\right)\left|\frac{d}{d u} \rho^{2}\right| \leq 2 \sup _{[0, u)}\left(\rho^{2} \frac{V^{\prime}}{V^{2}}+\frac{\rho^{2}}{\sqrt{V}}\right)+\int_{0}^{u}\left|\left(\frac{V^{\prime}}{V^{2}}+\frac{1}{\sqrt{V}}\right)^{\prime}\right| \rho^{2} .
$$

Finally, we can estimate the last integral by

$$
\begin{aligned}
\int_{0}^{u}\left|\left(\frac{V^{\prime}}{V^{2}}+\frac{1}{\sqrt{V}}\right)^{\prime}\right| \rho^{2} & \leq \sup _{[0, u)} \rho^{2} \operatorname{TV}_{[0, u)}\left(\frac{V^{\prime}}{V^{2}}+\frac{1}{\sqrt{V}}\right) \\
& =\sup _{[0, u)} \rho^{2}\left(\operatorname{TV}_{[0, u)} \frac{V^{\prime}}{V^{2}}+\sup _{[0, u)} \frac{1}{\sqrt{V}}\right)
\end{aligned}
$$

where in the last step we used the monotonicity of $V$.

\section{Elementary Properties of the Potential}

In this section we shall analyze the potential $V$ (2.3. 2.4) for large $\lambda$ and $\Omega$. More precisely, we consider the range

$$
\Omega>\Omega_{0} \quad \text { and } \quad \lambda>2 \Lambda \Omega
$$

for parameters $\Omega_{0}$ and $\Lambda$, which we can choose as large as we want. Then the potential looks qualitatively as in Figure 1. In the case $k \neq 0, V$ has a unique minimum $u_{0}$ given by

$$
\sin ^{2} u_{0}=\frac{1}{\Omega} \sqrt{k^{2}-\frac{1}{4}}
$$



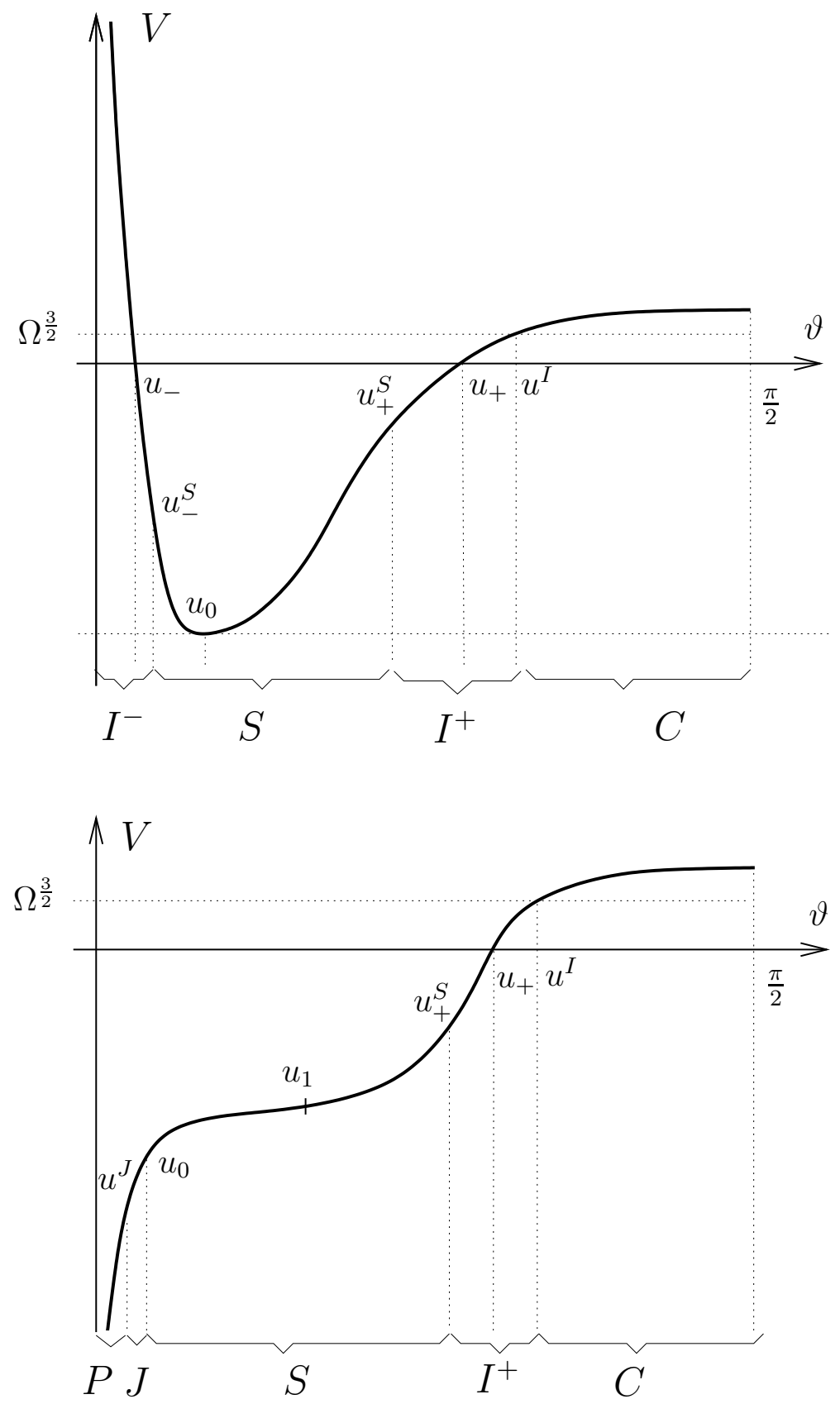

Figure 1: The potential $V$ in the cases $k \neq 0$ (top) and $k=0$ (bottom). 
and the potential is negative at the minimum,

$$
V\left(u_{0}\right)=\Omega\left(2 \sqrt{k^{2}-\frac{1}{4}}+2 k\right)-\lambda-\frac{1}{4}<-\Lambda \Omega
$$

(where in the last step we possibly increased $\Lambda$ ). $V$ is strictly decreasing on the interval $\left[0, u_{0}\right]$ and tends to infinity as $u \searrow 0$. Thus there is a unique $u_{-} \in\left[0, u_{0}\right]$ with $V\left(u_{-}\right)=0$. On the interval $\left[u_{0}, \frac{\pi}{2}\right], V$ is strictly increasing. Thus there is at most one $u_{+} \in\left(u_{0}, \frac{\pi}{2}\right]$ with $V\left(u_{+}\right)=0$. If no such $u_{+}$exists, we set $u_{+}=\frac{\pi}{2}$. For a given parameter $\kappa>0$ (which will be specified later) we set $\Delta u=\kappa / \sqrt{\Lambda \Omega}$. It is easily verified that by choosing $\Lambda$ sufficiently large we can arrange that $V\left(u_{0} \pm \Delta u\right)<0$. As a consequence, $\left|u_{ \pm}-u_{0}\right| \geq \Delta u$ and thus, using (6.3),

$$
\left|V\left(u_{0}\right)\right|\left|u_{ \pm}-u_{0}\right|^{2}>\kappa^{2} .
$$

Using monotonicity, we can thus uniquely introduce points $u_{+}^{S} \in\left(u_{0}, u_{+}\right)$and $u_{-}^{S} \in$ $\left(u_{-}, u_{0}\right)$ by the condition that

$$
\left|V\left(u_{ \pm}^{S}\right)\right|\left|u_{ \pm}-u_{ \pm}^{S}\right|^{2}=\kappa^{2}
$$

Finally, we introduce the point $u^{I} \in\left(u_{+}, \frac{\pi}{2}\right]$ by the condition $V\left(u^{I}\right)=\Omega^{\frac{3}{2}}$. If no such point exists, we set $u^{I}=\frac{\pi}{2}$.

In the case $k=0, V$ is monotone increasing on the whole interval $\left(0, \frac{\pi}{2}\right]$. We set

$$
u^{J}=\frac{1}{8 \sqrt{\lambda} \log ^{2} \lambda}, \quad u_{0}=\frac{\kappa}{\sqrt{\lambda}} \quad \text { and } \quad u_{1}=\frac{1}{\sqrt{\Omega}} .
$$

The points $u_{+}, u_{+}^{S} \in\left(u_{1}, u_{+}\right)$and $u^{I}$ are introduced as in the case $k \neq 0$.

We consider on $\left(0, \frac{\pi}{2}\right]$ the solution $y$ of the complex Riccati equation (3.4) with initial condition

$$
y\left(u_{0}\right)=i \sqrt{\left|V\left(u_{0}\right)\right|}-\frac{V^{\prime}\left(u_{0}\right)}{4 V\left(u_{0}\right)} .
$$

The next lemmas make the following statements precise: The intervals $S$ (as introduced in Figure 10 are "semiclassical" in the sense that Theorem 4.2 applies. On $C$ we can use the convexity estimate of Lemma 5.2 The interval $P$ near the pole can be treated by Theorem 4.4. Finally, the "intermediate regions" $I_{ \pm}$and $J$ can be controlled with Theorem 4.3 and Lemma 5.1

Lemma 6.1 For every $\delta>0$ and $k \in \mathbb{Z}$ there are parameters $\kappa, \Lambda, \Omega_{0}>0$ such that for all $\Omega, \lambda$ in the range (6.1), the quantity $K$ as defined by (4.16) is on the interval $S$ bounded by

$$
K \leq \delta
$$

Proof. The third derivative of $V$ can be written in the form

$$
\left.V^{\prime \prime \prime}=\frac{\cos u}{\sin ^{5} u} \text { (polynomial in } \sin ^{2} u \text { of degree } 3\right) \text {. }
$$

Hence $V^{\prime \prime \prime}$ has on any interval $[u, v] \subset\left[0, \frac{\pi}{2}\right]$ at most 4 zeros. Thus, after splitting up $[u, v]$ into at most four subintervals, $V^{\prime \prime \prime}$ has on each subinterval a fixed sign. On any such subinterval $[\underline{u}, \underline{v}]$ we can apply the estimate

$$
\int_{\underline{u}}^{\underline{v}}\left|V^{\prime \prime \prime}\right| d u \leq\left|V^{\prime \prime}(\underline{u})\right|+\left|V^{\prime \prime}(\underline{v})\right| .
$$


This makes it possible to control the total variation of $V^{\prime \prime}$ in (4.16) by $8 \sup \left|V^{\prime \prime}\right|$. We conclude that it suffices to show that on the interval $I$ the following two inequalities hold,

$$
\begin{aligned}
& \frac{V^{\prime 2}}{|V|^{3}} \leq \delta \\
& \frac{\left|V^{\prime \prime}\right|}{V^{2}} \leq \delta .
\end{aligned}
$$

We treat three cases separately.

First case: $k=0$ and $u_{+} \geq \frac{3 \pi}{8}$.

On the interval $\left[\frac{5 \pi}{16}, \frac{\pi}{2}\right]$, the potential $V$ is concave; more precisely,

$$
-\Omega^{2} \leq V^{\prime \prime} \leq-\frac{\Omega^{2}}{4}
$$

Integration yields for all $\tau \in\left(\frac{5 \pi}{16}, u_{+}\right]$the following bounds for $V^{\prime}$ and $V$,

$$
\begin{aligned}
\frac{\Omega^{2}}{4}\left(\frac{\pi}{2}-\tau\right) & \leq V^{\prime}(\tau) \leq \Omega^{2}\left(\frac{\pi}{2}-\tau\right) \\
V\left(u_{+}\right)-V(\tau) & \geq\left.\frac{\Omega^{2}}{8}\left(\frac{\pi}{2}-t\right)^{2}\right|_{u_{+}} ^{\tau}=\frac{\Omega^{2}}{8}\left(u_{+}-\tau\right)\left(\pi-u_{+}-\tau\right) .
\end{aligned}
$$

Since $V\left(u_{+}\right)$is either zero or negative, it follows that

$$
|V(\tau)| \geq \frac{\Omega^{2}}{8}\left(u_{+}-\tau\right)\left(\pi-u_{+}-\tau\right) .
$$

Combining the inequalities (6.9, 6.10, 6.11), we obtain for all $\tau \in\left(\frac{5 \pi}{16}, u_{+}^{S}\right]$ the estimates

$$
\begin{aligned}
& \frac{V^{\prime}(\tau)^{2}}{|V(\tau)|^{3}} \leq 8^{3} \frac{\Omega^{4}\left(\frac{\pi}{2}-\tau\right)^{2}}{\Omega^{6}\left(u_{+}-\tau\right)^{3}\left(\pi-u_{+}-\tau\right)^{3}} \leq \frac{8^{3}}{\Omega^{2}\left(u_{+}-u_{+}^{S}\right)^{4}} \\
& \frac{\left|V^{\prime \prime}(\tau)\right|}{V^{2}(\tau)} \leq 64 \frac{\Omega^{2}}{\Omega^{4}\left(u_{+}-\tau\right)^{2}\left(\pi-u_{+}-\tau\right)^{2}} \leq \frac{64}{\Omega^{2}\left(u_{+}-u_{+}^{S}\right)^{4}} .
\end{aligned}
$$

In order to estimate the factor $\left(u_{+}-u_{+}^{S}\right)$ from below, we use (6.11) in the defining equation for $u_{+}^{S}$, (6.4),

$$
\frac{\Omega^{2}}{8}\left(u_{+}-u_{+}^{S}\right)^{4} \leq\left|V\left(u_{+}^{S}\right)\right|\left(u_{+}-u_{+}^{S}\right)^{2}=\kappa^{2} .
$$

Using this inequality in (6.12, 6.13) and choosing $\kappa$ sufficiently large, we obtain 6.7 6.8 for all $\tau \in\left(\frac{5 \pi}{16}, u_{+}^{S}\right]$.

On the interval $\left[u_{0}, u_{1}\right]$, a short calculation using (2.3, 6.5, 6.1) shows that

$$
|V| \geq \frac{\lambda}{2}, \quad\left|V^{\prime}\right|^{2} \leq \frac{\lambda^{3}}{\kappa^{6}}, \quad\left|V^{\prime \prime}\right| \leq \frac{2 \lambda^{2}}{\kappa^{4}} \quad \text { on }\left[u_{0}, u_{1}\right],
$$

again proving (6.7, 6.8).

On the remaining interval $\left(u_{1}, \frac{5 \pi}{16}\right)$, we know from the monotonicity of the potential and (6.11) that

$$
|V(\tau)| \geq \frac{\Omega^{2}}{8}\left(u_{+}-\frac{5 \pi}{16}\right)\left(\pi-u_{+}-\frac{5 \pi}{16}\right) \geq \frac{\pi^{2}}{2048} \Omega^{2} .
$$


Furthermore, a short calculation using (2.3) shows that on $\left(u_{1}, \frac{5 \pi}{16}\right)$

$$
\left|V^{\prime}\right|+\left|V^{\prime \prime}\right| \leq 4 \Omega^{2} \quad \text { on }\left[u_{1}, \frac{\pi}{2}\right]
$$

We conclude that, choosing $\Omega_{0}$ sufficiently large, we can again arrange that (6.7 6.8) holds.

Second case: $k=0$ and $u_{+}<\frac{3 \pi}{8}$.

On the interval $\left[u_{0}, u_{1}\right]$, we can again use the estimate (6.14). Conversely, on the interval $\left[u_{1}, \frac{3 \pi}{8}\right]$, a short calculation shows that $V^{\prime \prime}$ can be bounded in terms of higher powers of the first derivatives; more precisely,

$$
\left|V^{\prime \prime}\right| \leq 10\left|V^{\prime}\right|^{\frac{4}{3}}
$$

This inequality allows us to deduce (6.8) from (6.7). Hence it remains to prove the inequality (6.7) on the interval $\left[u_{1}, u_{+}^{S}\right]$.

On the interval $\left[u_{1}, u_{+}^{S}\right]$, the potential $V$ is either convex or else at least the second derivative of $V$ is large compared to $\left|V^{\prime}\right|^{\frac{4}{3}}$. More precisely, by choosing $\Omega_{0}$ sufficiently large, we can arrange that

$$
V^{\prime \prime} \geq-\kappa^{-\frac{2}{3}} V^{\prime \frac{4}{3}} \text {. }
$$

We shall derive an upper bound for $\Delta u:=u_{+}-u_{+}^{S}$; for ease in notation the subscript ' + ' will be omitted. We rewrite (6.16) as

$$
\frac{d}{d u}\left(V^{\prime-\frac{1}{3}}\right)=-\frac{1}{3} \frac{V^{\prime \prime}}{V^{\prime \frac{4}{3}}} \leq \frac{\kappa^{-\frac{2}{3}}}{3} .
$$

We integrate from $u^{S}$ to $\tau \in\left[u^{S}, u\right]$ to obtain

$$
V^{\prime}(\tau) \geq\left(V^{\prime}\left(u^{S}\right)^{-\frac{1}{3}}+\frac{\kappa^{-\frac{2}{3}}}{3}\left(\tau-u^{S}\right)\right)^{-3}
$$

Integrating $\tau$ over the interval $\left[u^{S}, u\right]$, we obtain for $\Delta V:=V(u)-V\left(u^{S}\right)$ the estimate

$$
\Delta V \geq \frac{3 \kappa^{\frac{2}{3}}}{2} V^{\prime}\left(u^{S}\right)^{\frac{2}{3}}\left(1-\frac{1}{(1+\alpha)^{2}}\right) \quad \text { with } \quad \alpha:=\frac{\kappa^{-\frac{2}{3}}}{3} V^{\prime}\left(u^{S}\right)^{\frac{1}{3}} \Delta u .
$$

The inequality

$$
1-\frac{1}{(1+\alpha)^{2}} \geq \frac{\alpha}{1+\alpha}
$$

gives

$$
\Delta V \geq \frac{V^{\prime}\left(u^{S}\right)}{2} \frac{\Delta u}{1+\alpha} .
$$

By definition of $u^{S}$, (6.4), we know that $\Delta V \cdot(\Delta u)^{2}=\kappa^{2}$. Hence, multiplying the last inequality by $(\Delta u)^{2}$, we obtain

$$
\kappa^{2} \geq \frac{V^{\prime}\left(u^{S}\right)}{2} \frac{(\Delta u)^{3}}{1+\alpha}
$$

Using the definition of $\alpha$ gives the inequality

$$
(\Delta u)^{3}-\frac{2}{3}\left(\kappa^{\frac{2}{3}} V^{\prime}\left(u^{S}\right)^{-\frac{1}{3}}\right)^{2} \Delta u-2\left(\kappa^{\frac{2}{3}} V^{\prime}\left(u^{S}\right)^{-\frac{1}{3}}\right)^{3} \leq 0 .
$$


Since the polynomial $x^{3}-\frac{2 x}{3}-2$ is positive for $x \geq 2$, we conclude that

$$
\Delta u \leq 2 \kappa^{\frac{2}{3}} V^{\prime}\left(u^{S}\right)^{-\frac{1}{3}} .
$$

Using again the relation $\Delta V(\Delta u)^{2}=\kappa^{2}$, we get an upper bound for $\Delta V$,

$$
\Delta V \geq \frac{\kappa^{\frac{2}{3}}}{4} V^{\prime}\left(u^{S}\right)^{\frac{2}{3}}
$$

This proves the inequality (6.7) at $u=u^{S}$.

Next we shall show that (6.7) holds on the whole interval $\left(u_{1}, u^{S}\right]$. To this end, we introduce on this interval the function $f$ by

$$
f=V^{\prime 2}+\frac{4^{3}}{\kappa^{2}} V^{3} .
$$

We saw above that $f\left(u^{S}\right)<0$; our goal is to show that $f \leq 0$ on $\left(u_{1}, u^{S}\right]$. Let $\left(v, u^{S}\right]$ with $u_{1} \leq v<u^{S}$ be the maximal interval on which $f$ is negative. We apply (6.16) to obtain

$$
\begin{aligned}
f^{\prime}(v) & =V^{\prime}\left(2 V^{\prime \prime}+\frac{3 \cdot 4^{3}}{\kappa^{2}} V^{2}\right) \\
& \geq V^{\prime}\left(-2 \kappa^{-\frac{2}{3}} V^{\prime \frac{4}{3}}+\frac{3 \cdot 4^{3}}{\kappa^{2}} V^{2}\right) \geq 10 \kappa^{-\frac{2}{3}} V^{\prime \frac{7}{3}}>0,
\end{aligned}
$$

where in the last line we used that $f(v) \leq 0$. The last inequality contradicts the maximality of the interval $\left(v, u^{S}\right]$ unless $v=u_{1}$. This concludes the proof in the second case.

Third case: $k \neq 0$.

On the interval $\left(u_{-}^{S}, u_{0}\right]$, the proof of the second case goes through without changes if we consider the integral backwards and set $u_{1}=u_{0}-1 /(4 \sqrt{\Omega})$. On the remaining interval $\left[u_{0}, u_{+}^{S}\right]$, we can use the proof of the first case and the second case after setting $u_{1}=u_{0}+1 /(4 \sqrt{\Omega})$.

Lemma 6.2 For sufficiently large $\Lambda$ and $\Omega_{0}$, the parameter $L$ (as defined by 5.7)) is on the interval $C$ for all $\Omega, \lambda$ in the range (6.1) bounded by

$$
L \leq \frac{3}{\sqrt{\Omega_{0}}} .
$$

Proof. Similar to (6.9 6.10), one easily sees that $V^{\prime}$ and $V^{\prime \prime}$ satisfy on $C$ the bound $\left|V^{\prime}\right|+$ $\left|V^{\prime \prime}\right| \leq 4 \Omega^{2}$. On the other hand, it is clear from the definition of $u^{I}$ that $|V| \geq \Omega^{\frac{3}{2}}$ on $C$. This immediately gives the lemma.

Lemma 6.3 For sufficiently large $\Lambda$ and $\Omega_{0}$, the potential

$$
B(u):=V(u)+\frac{1}{4 u^{2}}
$$

satisfies on the interval $P$ the inequality

$$
\left(u^{J}\right)^{2}\left(1+\log ^{2} u^{J}\right)^{2}\|B\|_{\infty} \leq \frac{1}{8} .
$$


Proof. A short calculation shows that $B$ is bounded from above by

$$
|B(u)| \leq \Omega^{2} u^{2}+2 \lambda .
$$

From the definition of $u^{J}$, (6.5), it is clear that for large $\lambda,\left|\log u^{J}\right| \leq \log \lambda$, and thus

$$
\begin{aligned}
\left(u^{J}\right)^{2}\left(1+\log ^{2} u^{J}\right)^{2}\|B\|_{\infty} & \leq \frac{1}{64 \lambda \log ^{4} \lambda}\left(1+\log ^{2} \lambda\right)^{2}\|B\|_{\infty} \\
& \leq \frac{1}{32 \lambda}\|B\|_{\infty} \stackrel{6.18}{\leq} \frac{1}{32 \lambda}\left(\frac{\Omega^{2}}{\lambda}+2 \lambda\right) \leq \frac{1}{8}
\end{aligned}
$$

where in the last step we again used (6.1).

Lemma 6.4 For every $\delta>0$ and $k \in \mathbb{Z}$ there are parameters $\kappa, \Lambda, \Omega_{0}>0$ such that for all $\Omega, \lambda$ in the range (6.1),

$$
\left|I_{ \pm}\right| \leq \delta
$$

Proof. We choose $\Omega_{0}$ so large that $u_{0}<\frac{\delta}{4}$. Then clearly $\left|I_{-}\right| \leq \delta$. Furthermore, it is readily verified that the potential is increasing on the interval $K:=\left[\frac{\delta}{4}, \frac{\pi}{2}-\frac{\delta}{4}\right]$ at the following rate,

$$
V(v)-V(u) \geq c(v-u)^{2} \Omega^{2} \quad \text { for all } u, v \in K, v>u,
$$

where $c$ is independent of $\lambda$ and $\Omega$. This implies that

$$
\left|\left[u_{+}^{S}, u_{+}\right] \cap K\right| \leq c^{-\frac{1}{4}} \sqrt{\frac{\kappa}{\Omega}},
$$

because otherwise (6.4) would be violated. Furthermore, the condition $V\left(u^{I}\right) \leq \Omega^{\frac{3}{2}}$ implies that

$$
\left|\left[u_{+}, u^{I}\right] \cap K\right| \leq c^{-\frac{1}{2}} \Omega^{-\frac{1}{4}}
$$

We conclude that by increasing $\Omega_{0}$, we can arrange that $\left|I_{+} \cap K\right| \leq \frac{\delta}{2}$ and thus $\left|I_{+}\right| \leq \delta$.

\section{Spectral Estimates for the Selfadjoint Problem}

In this section we shall prove Lemma 2.1] We begin by reducing the problem to an estimate for $y_{\lambda}$.

Lemma 7.1 Assume that for any given $k \in \mathbb{Z}$ and $\varepsilon>0$, there are constants $\Lambda, \Omega_{0}>0$ such that

$$
\int_{0}^{\frac{\pi}{2}} \operatorname{Im} y_{\lambda} \leq \varepsilon
$$

for all $\Omega$ and $\lambda$ in the range [6.1). Then Lemma 2.1 holds. 
Proof. According to the asymptotics (2.9) it suffices to consider $\lambda$ in the range $\lambda>\Lambda \Omega$ for sufficiently large $\Lambda$. Let us consider the quotient $\operatorname{Re}(y) / \operatorname{Im}(y)$ in (3.10). According to Theorem 4.2 and Theorem 4.3. there is a constant $c>0$ such that for all $\Omega>\Omega_{0}$ and $\lambda>\Lambda \Omega$,

$$
\frac{\operatorname{Re} y}{\operatorname{Im} y}>-c \quad \text { on }\left[u_{0}, u_{+}\right]
$$

On the interval $\left[u_{+}, \frac{\pi}{2}\right], \rho^{2}$ is convex, and using the identity

$$
\frac{\operatorname{Re} y}{\operatorname{Im} y}=\frac{\rho^{2}}{w} \operatorname{Re} y=\frac{1}{2 w}\left(\rho^{2}\right)^{\prime}
$$

one sees that $\operatorname{Re}(y) / \operatorname{Im}(y)$ is monotone increasing. We conclude that the inequality (7.2) also holds at $u=\frac{\pi}{2}$, and thus

$$
-\frac{\pi}{2}<-\arctan c<\arctan \left(\frac{\operatorname{Re} y\left(\frac{\pi}{2}\right)}{\operatorname{Im} y\left(\frac{\pi}{2}\right)}\right)<\frac{\pi}{2} .
$$

Using the last bounds in (3.10) one sees that for two neighboring eigenvalues, the phases must differ at least by $\delta:=\frac{\pi}{2}-\arctan c$,

$$
\varphi_{n+1}-\left.\varphi_{n}\right|_{0} ^{\frac{\pi}{2}} \geq \delta
$$

From (3.11) one sees that this inequality is also true for the states of odd parity (with $\delta=$ $\pi$ ). Applying (3.7) and the mean value theorem, we conclude that there is $\lambda \in\left[\lambda_{n}^{ \pm}, \lambda_{n+1}^{ \pm}\right]$ such that

$$
\left(\lambda_{n+1}^{ \pm}-\lambda_{n}^{ \pm}\right) \int_{0}^{\frac{\pi}{2}} \operatorname{Im} y_{\lambda} \geq \delta .
$$

Hence the upper bound (7.1) gives the desired gap estimate.

We establish (17.1) by deriving separate estimates in the regions $S, I_{ \pm}, C$ and near the pole.

Lemma 7.2 For any given $k \in \mathbb{Z}$ and $\varepsilon>0$, there are constants $\Lambda, \Omega_{0}>0$ such that on the interval $S$,

$$
\left|y_{\lambda}\right| \leq \varepsilon .
$$

Proof. Differentiating the initial condition (6.6) gives

$$
y_{\lambda}\left(u_{0}\right)=\frac{i}{2 \sqrt{\left|V\left(u_{0}\right)\right|}}+\frac{V^{\prime}\left(u_{0}\right)}{4 V^{2}\left(u_{0}\right)} .
$$

This can be estimated using Lemma 6.1,

$$
\left|y_{\lambda}\left(u_{0}\right)\right| \leq \frac{1}{2 \sqrt{\left|V\left(u_{0}\right)\right|}}\left(1+\frac{\left|V^{\prime}\left(u_{0}\right)\right|}{\left|V\left(u_{0}\right)\right|^{\frac{3}{2}}}\right) \leq \frac{1}{\sqrt{\left|V\left(u_{0}\right)\right|}} .
$$

For given $u \in S$, we compute $y_{\lambda}(u)$ via (3.13). This gives rise to the estimate

$$
\begin{aligned}
\left|y_{\lambda}(u)\right| & \leq \frac{\rho^{2}\left(u_{0}\right)}{\rho^{2}(u)}\left|y_{\lambda}\left(u_{0}\right)+\frac{1}{2 y\left(u_{0}\right)}\right|+\frac{1}{2|y(u)|}+\frac{1}{\rho^{2}(u)} \int_{u_{0}}^{u} \frac{\left|V-y^{2}\right|}{2|y|^{2}} \rho^{2} \\
& \leq \frac{2 \operatorname{Im} y(u)}{\left|V\left(u_{0}\right)\right|}+\frac{1}{2|y(u)|}+\operatorname{Im} y(u) \int_{u_{0}}^{u} \frac{\left|V-y^{2}\right|}{2|y|^{2}} \frac{1}{\operatorname{Im} y(u)}
\end{aligned}
$$


where in the last step we used (7.4, 6.4 3.8). According to Lemma 6.1 we can apply Theorem 4.2. We thus obtain for any $K \in(0,1)$ the estimates

$$
\begin{aligned}
|y| & \leq 22 \sqrt{|V|} \\
|y-i \sqrt{|V|}| & \leq \sqrt{|V|}(20 K+\sqrt{K}) \leq 21 \sqrt{K} \sqrt{|V|} \\
\left|V-y^{2}\right| & =|(y-i \sqrt{|V|})(y+i \sqrt{|V|})| \\
& \leq 21 \sqrt{K} \sqrt{|V|} 23 \sqrt{|V|} \leq 500|V| \sqrt{K}
\end{aligned}
$$

and, after choosing $K<1 / 42$, the inequality (17.7) shows that

$$
\operatorname{Im} y \geq \frac{\sqrt{|V|}}{2}
$$

Using the inequalities (7.6, 17.9) in (7.5) gives

$$
\left|y_{\lambda}(u)\right| \leq \frac{44 \sqrt{|V(u)|}}{\left|V\left(u_{0}\right)\right|}+\frac{1}{\sqrt{|V(u)|}}+22 \sqrt{|V(u)|} \int_{u_{0}}^{u} \frac{\left|V-y^{2}\right|}{|y|^{2}} \frac{1}{\sqrt{|V|}},
$$

and, since $V$ is monotonous, it follows using (7.9, (7.8) that

$$
\begin{aligned}
\left|y_{\lambda}(u)\right| & \leq \frac{45}{\sqrt{|V(u)|}}+22 \int_{u_{0}}^{u} \frac{\left|V-y^{2}\right|}{|y|^{2}} \\
& \leq \frac{45}{\sqrt{|V(u)|}}+88 \cdot 500 \sqrt{K} \frac{\pi}{2} .
\end{aligned}
$$

The last expression can be made arbitrarily small according to Lemma 6.1

Lemma 7.3 For any given $k \in \mathbb{Z}$ and $\varepsilon>0$, there are constants $\Lambda, \Omega_{0}>0$ such that on the intervals $I_{ \pm}$,

$$
\left|y_{\lambda}\right| \leq \varepsilon .
$$

Proof. We only consider the interval $I_{+}$; the proof for $I_{-}$is analogous. For any $v \in$ $\left(u_{+}^{S}, u_{+}\right]$, we compute $y_{\lambda}$ via (3.12) with $u=u_{+}^{S}$,

$$
y_{\lambda}(v)=-\frac{z^{2}\left(u_{+}^{S}\right) y_{\lambda}\left(u_{+}^{S}\right)}{z^{2}(v)}-\frac{1}{z^{2}(v)} \int_{u_{+}^{S}}^{v} z^{2} .
$$

According to the definition of $u_{+}^{S}$, (6.4), we can apply Theorem4.3. This gives the estimate

$$
\left|y_{\lambda}(v)\right| \leq\left|y_{\lambda}\left(u_{+}^{S}\right)\right| \frac{\operatorname{Im} y(v)}{\operatorname{Im} y\left(u_{+}^{S}\right)}+\operatorname{Im} y(v) \int_{u_{+}^{S}}^{v} \frac{1}{\operatorname{Im} y} \leq c_{2}^{2}\left|y_{\lambda}\left(u_{+}^{S}\right)\right|+c_{2}^{2}\left(v-u_{+}^{S}\right) .
$$

This can be made arbitrarily small according to Lemma 7.2 and Lemma 6.4.

If $v \in\left(u_{+}, u^{I}\right]$, the change of $y_{\lambda}$ on the interval $\left(u_{+}, v\right)$ can be estimated similarly using Lemma 5.1 . 
Lemma 7.4 For any given $k \in \mathbb{Z}$ and $\varepsilon>0$, there are constants $\Lambda, \Omega_{0}>0$ such that on the interval $C$,

$$
\left|y_{\lambda}\right| \leq \epsilon .
$$

Proof. We again compute $y_{\lambda}$ via (3.12). This gives for any $v \in C$ the estimate

$$
\left|y_{\lambda}(v)\right| \leq \frac{\rho^{2}\left(u^{I}\right)}{\rho^{2}(v)}\left|y_{\lambda}\left(u^{I}\right)\right|+\frac{1}{\rho^{2}(v)} \int_{u^{I}}^{v} \rho^{2} .
$$

The first summand can be made arbitrarily small according to Lemma 7.3 and Lemma 5.1 . whereas the second summand can be handled with Lemma 5.2 and Lemma 6.2 .

Lemma 7.5 For any given $k \in \mathbb{Z}$ and $\varepsilon>0$, there are constants $\Lambda, \Omega_{0}>0$ such that for all $\lambda$ and $\Omega$ in the range (6.1),

$$
\int_{P \cup J}\left|y_{\lambda}\right| \leq \epsilon
$$

Proof. A short calculation using (2.3, 6.5) shows that on the interval $J$,

$$
\frac{\lambda}{8 \kappa^{2}} \leq\left|V\left(u_{0}\right)\right| \leq \frac{\lambda}{\kappa^{2}}, \quad 8 \lambda \log ^{4} \lambda \leq\left|V\left(u^{J}\right)\right| \leq 64 \lambda \log ^{4} \lambda, \quad|J| \leq \frac{\kappa}{\sqrt{\lambda}} .
$$

In particular, $\left|V\left(u_{0}\right)\right||J|^{2} \leq 1$, and so we can apply Theorem 4.3 to obtain on $J$ the estimates

$$
|y| \leq 16 c_{2} \sqrt{\lambda} \log ^{2} \lambda, \quad \operatorname{Im} y \geq \frac{1}{16 c_{2} \kappa^{2}} \frac{\sqrt{\lambda}}{\log ^{2} \lambda} .
$$

These estimates allow us to bound $y_{\lambda}$ on $J$ again using (3.12). Namely, for all $v \in J$,

$$
\left|y_{\lambda}(v)\right| \leq\left|y_{\lambda}\left(u_{0}\right)\right| \frac{\operatorname{Im} y(v)}{\operatorname{Im} y\left(u_{0}\right)}+\operatorname{Im} y(v) \int_{v}^{u_{0}} \frac{1}{\operatorname{Im} y} .
$$

Estimating the factor $\left|y_{\lambda}\left(u_{0}\right)\right|$ by (17.4), we obtain

$$
\left|y_{\lambda}(v)\right| \leq c_{3} \frac{\log ^{4} \lambda}{\sqrt{\lambda}} \quad \text { on } J
$$

with $c_{3}=256 c_{2} \kappa^{3}$. By increasing $\Lambda$ this can be made arbitrarily small.

On the interval $P$, we apply Lemma 4.4 with $C=64 c_{2} \kappa^{2} \log ^{4} \lambda$. This gives the estimate

$$
\frac{1}{c_{4} \log ^{p} \lambda} \frac{1}{v \log ^{2} v} \leq \operatorname{Im} y(v) \leq \frac{c_{4} \log ^{p} \lambda}{v \log ^{2} v}
$$

with $p=14$ and a constant $c_{4}$ which is independent of $\Lambda$ and $\Omega$. We again estimate $y_{\lambda}$ using (3.12),

$$
\left|y_{\lambda}(v)\right| \leq\left|y_{\lambda}\left(u^{J}\right)\right| \frac{\operatorname{Im} y(v)}{\operatorname{Im} y\left(u^{J}\right)}+\operatorname{Im} y(v) \int_{v}^{u^{J}} \frac{1}{\operatorname{Im} y} .
$$

Estimating $y\left(u^{J}\right)$ and $y_{\lambda}\left(u^{J}\right)$ by (7.10, 7.11) and using (7.12) we get for all $v \in P$,

$$
\left|y_{\lambda}(v)\right| \leq c_{5} \frac{\log ^{p+6} \lambda}{\lambda} \frac{1}{v \log ^{2} v}+c_{5} \frac{\log ^{2 p} \lambda}{v \log ^{2} v} \int_{v}^{u^{J}} \tau \log ^{2} \tau d \tau
$$


for a suitable constant $c_{5}$. This expression is not bounded as $v \searrow 0$. But the pole is integrable, and the calculation

$$
\begin{aligned}
\int_{0}^{u^{J}} \frac{d v}{v \log ^{2} v} & =-\frac{1}{\log u^{J}} \\
\int_{0}^{u^{J}} \frac{d v}{v \log ^{2} v} \int_{v}^{u^{J}} \tau \log ^{2} \tau d \tau & =\int_{0}^{u^{J}} d \tau \tau \log ^{2} \tau \int_{0}^{\tau} \frac{d v}{v \log ^{2} v} \\
& =-\int_{0}^{u^{J}} \tau \log \tau d \tau=\frac{1}{4}\left(u^{J}\right)^{2}\left(1-2 \log u^{J}\right)
\end{aligned}
$$

shows that, by increasing $\Lambda$, we can make the resulting integrals over $P$ arbitrarily small.

This completes the proof of Theorem 1.3

\section{Slightly Non-Selfadjoint Perturbations}

It remains to prove Theorem 1.1 In preparation, we split up the spheroidal wave operator as

$$
\mathcal{A}=\mathcal{A}_{0}+W
$$

with

$$
\begin{aligned}
& \mathcal{A}_{0}=-\frac{d}{d \cos \vartheta} \sin ^{2} \vartheta \frac{d}{d \cos \vartheta}+\frac{1}{\sin ^{2} \vartheta}\left(\operatorname{Re} \Omega \sin ^{2} \vartheta+k\right)^{2} \\
& W=2 i \operatorname{Im} \Omega\left(\operatorname{Re} \Omega \sin ^{2} \vartheta+k\right)-(\operatorname{Im} \Omega)^{2} \sin ^{2} \vartheta .
\end{aligned}
$$

The symmetric operator $\mathcal{A}_{0}$ clearly satisfies the hypothesis of Theorems 1.2 and 1.3 , whereas the complex potential $W$ is uniformly bounded according to assumption (1.5),

$$
|W| \leq 2(k+1) c+c^{2}=: \frac{\rho}{2}
$$

Our method is to treat $W$ as a slightly non-selfadjoint perturbation as introduced by Kato [8, V.4.5]; see in particular [8, Theorem 4.15a]. Unfortunately, the latter theorem is not quite strong enough for our purpose. For clarity, we here repeat the basic ideas of Kato and give a detailed proof of our main theorem.

Proof of Theorem 1.1. Throughout the proof, we restrict all operators either to $\mathcal{H}_{+}$ or $\mathcal{H}_{-}$. Applying Theorems 1.2 and 1.3 to the operator $\mathcal{A}_{0}$ and $\gamma=8 \rho$, we obtain for the eigenvalues $0 \leq \lambda_{1}<\lambda_{2}<\cdots$ of $\mathcal{A}_{0}$ the estimates

$$
\lambda_{n+1}-\lambda_{n}>\gamma \quad \text { for all } n \geq N \text { and } \Omega \in \mathbb{R} .
$$

For all $\lambda \notin \sigma\left(\mathcal{A}_{0}\right)$, the resolvent $R_{\lambda}^{0}:=\left(\lambda-\mathcal{A}_{0}\right)^{-1}$ exists and satisfies the bound

$$
\left\|R_{\lambda}^{0}\right\| \leq \frac{1}{\operatorname{dist}\left(\lambda, \sigma\left(\mathcal{A}_{0}\right)\right)}
$$

Since the spectrum of $\mathcal{A}_{0}$ is real, we have in particular

$$
\left\|R_{\lambda}^{0}\right\| \leq \frac{1}{|\operatorname{Im} \lambda|}
$$




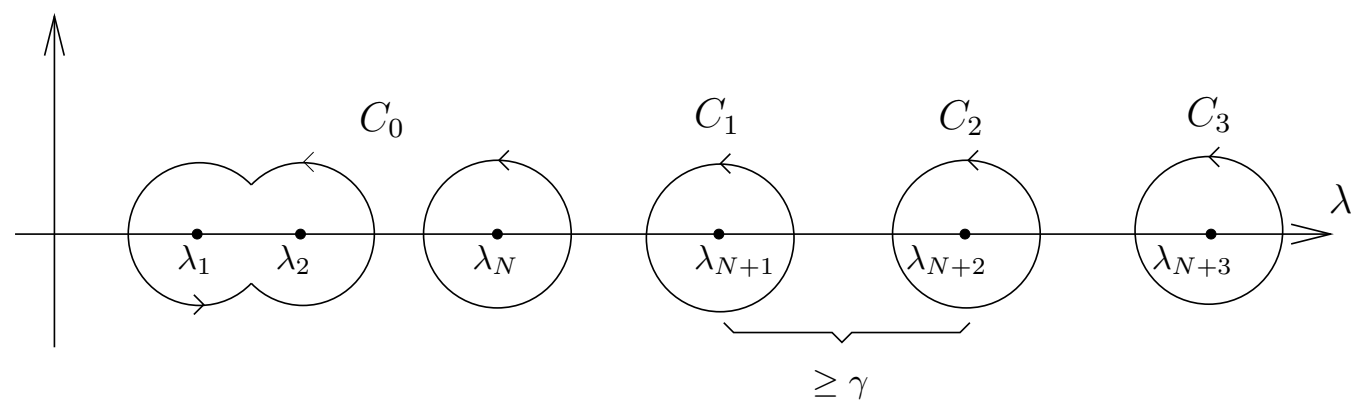

Figure 2: The contours $C_{k}$.

Around each $\lambda_{n}$, we draw a circle of radius $\rho$. The first $N$ circles may intersect, and we take the outermost lines to define the contour $C_{0}$,

$$
C_{0}=\partial\left(B_{\rho}\left(\lambda_{1}\right) \cup \ldots \cup B_{\rho}\left(\lambda_{N}\right) .\right.
$$

All the following circles do not intersect and give rise to the contours

$$
C_{k}=\partial B_{\rho}\left(\lambda_{N+k}\right), \quad k \geq 1
$$

(see Figure 21). Since the distance of these contours to the spectral points of $\mathcal{A}_{0}$ is at least $\rho$, we have for $\lambda$ on any of these contours,

$$
\left\|R_{\lambda}^{0} W\right\| \leq\left\|R_{\lambda}^{0}\right\|\|W\| \leq \frac{1}{2}
$$

Hence the operator $1+R_{\lambda}^{0} W$ is invertible with a Neumann series. We conclude that the resolvent $R_{\lambda}:=(\lambda-\mathcal{A})^{-1}=\left(\left(\lambda-\mathcal{A}_{0}\right)\left(1-R_{\lambda}^{0} W\right)\right)^{-1}=\left(1-R_{\lambda}^{0} W\right)^{-1} R_{\lambda}^{0}$ exists and

$$
\left\|R_{\lambda}\right\| \leq 2\left\|R_{\lambda}^{0}\right\|
$$

This allows us to introduce the operators $Q_{k}$ as the following contour integrals

$$
Q_{k}=\frac{1}{2 \pi i} \oint_{C_{k}} R_{\lambda} d \lambda
$$

The Cauchy integral formula together with the resolvent identity

$$
R_{\lambda} R_{\lambda^{\prime}}=-\frac{1}{\lambda-\lambda^{\prime}}\left(R_{\lambda}-R_{\lambda^{\prime}}\right)
$$

immediately yield that the operators $Q_{k}$ are projectors onto invariant subspaces of $\mathcal{A}$, and that they are holomorphic in $\Omega$. Furthermore, they are uniformly bounded because according to 8.3 8.2 and the definition of the contours,

$$
\left\|Q_{k}\right\| \leq \frac{1}{2 \pi} \oint_{C_{k}} 2\left\|R_{\lambda}^{0}\right\| \leq 2 N
$$

We introduce the operators $P_{K}$ as the finite sums

$$
P_{K}=\sum_{k=0}^{K} Q_{k} .
$$




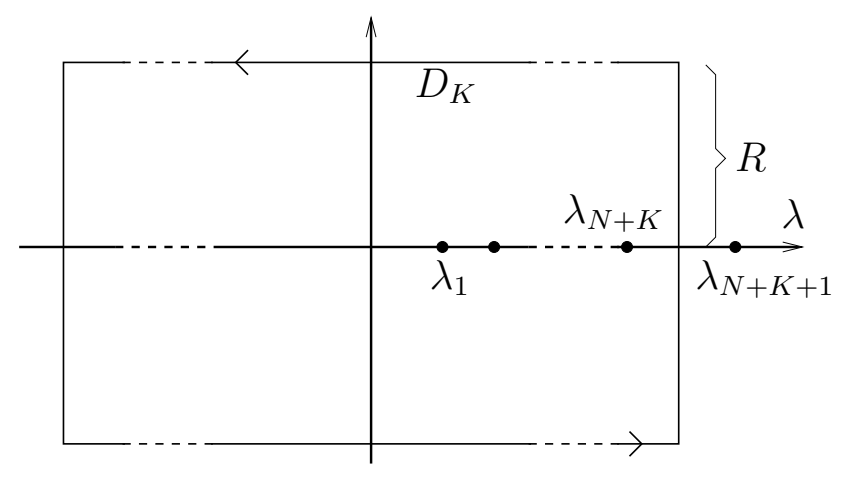

Figure 3: The contours $D_{K}$.

For the unperturbed operator $\mathcal{A}_{0}$, we introduce similarly the projectors $Q_{k}^{0}$ and $P_{K}^{0}$. Let us derive estimates for the difference $P_{K}-P_{K}^{0}$. We first write it as the contour integral

$$
P_{K}-P_{K}^{0}=\frac{1}{2 \pi i} \oint_{D_{K}}\left(R_{\lambda}-R_{\lambda}^{0}\right) d \lambda
$$

where $D_{K}$ is a rectangle with side lengths $\lambda_{N+K}+\lambda_{N+K+1}$ and $2 R$ centered at the origin (see Figure 3). Since $\operatorname{dist}\left(D_{k}, \sigma\left(\mathcal{A}_{0}\right)\right)>\rho$, the inequality (8.3) again holds. Using the resolvent identity

$$
R_{\lambda}-R_{\lambda}^{0}=R_{\lambda} W R_{\lambda}^{0}
$$

together with (8.1, 8.2), we obtain for any $\lambda$ on the contour $D_{K}$ for sufficiently large $R$ the estimate

$$
\left\|R_{\lambda}-R_{\lambda}^{0}\right\| \leq \frac{8 \rho}{\gamma^{2}+4(\operatorname{Im} \lambda)^{2}} .
$$

This inequality allows us to take in (8.4) the limit $R \rightarrow \infty$ to obtain the estimate

$$
\left\|P_{K}-P_{K}^{0}\right\| \leq \frac{4 \rho}{\gamma}=\frac{1}{2} .
$$

This estimate can be improved if the operator $P_{K}-P_{K}^{0}$ is restricted to the range of $P_{L}^{0}$, $L<K$. Namely, applying the bound

$$
\left\|R_{\lambda}^{0} P_{L}^{0}\right\| \leq \max _{n=1, \ldots, N+L}\left|\lambda-\lambda_{n}\right|^{-1}
$$

to the resolvent identity gives for any $\lambda$ on the contour $D_{K}$ for sufficiently large $R$ the bound

$$
\left\|\left(P_{K}-P_{K}^{0}\right) P_{L}^{0}\right\| \leq \frac{8 \rho}{|\gamma+2 i \operatorname{Im} \lambda|\left|\lambda-\lambda_{N+L}\right|} .
$$

Substituting this estimate into the contour integral, taking the limit $R \rightarrow \infty$ and estimating the resulting integral as follows,

$$
\int_{0}^{\infty} \frac{d x}{\sqrt{a^{2}+x^{2}} \sqrt{b^{2}+x^{2}}} \leq \int_{0}^{\sqrt{a b}} \frac{d x}{a b}+\int_{\sqrt{a b}}^{\infty} \frac{d x}{x^{2}} \leq \frac{2}{\sqrt{a b}}
$$

we conclude that

$$
\left\|\left(P_{K}-P_{K}^{0}\right) P_{L}^{0}\right\| \leq \frac{8 \rho}{\sqrt{\gamma\left(\lambda_{N+K}-\lambda_{N+L}\right)}}, \quad L<K
$$


The inequality (8.5) allows us to determine the rank of the operators $P_{K}$. Namely, for every $\Psi$ in the range of $P_{K}^{0}$,

$$
\left\|P_{K} \Psi\right\| \geq\left\|P_{K}^{0} \Psi\right\|-\left\|\left(P_{K}-P_{K}^{0}\right) \Psi\right\| \geq \frac{1}{2}\|\Psi\| .
$$

In particular, $\Psi$ is not in the kernel of $P_{K}$. This shows that the rank of $P_{K}$ is greater or equal to the rank of $P_{K}^{0}$. Interchanging the roles of $P_{K}$ and $P_{K}^{0}$, we see that $P_{K}$ and $P_{K}^{0}$ have the same rank. Since $P_{K}^{0}$ is the projector on the eigenspaces of $\mathcal{A}_{0}$ corresponding to the eigenvalues $\lambda_{1}, \ldots, \lambda_{N+K}$, the dimension of its range is $N+k$. We conclude that $Q_{0}$ is a projector on an $N$-dimensional invariant subspace of $\mathcal{A}$ and $Q_{1}, Q_{2}, \ldots$ are projectors on 1-dimensional eigenspaces.

The inequalities (8.5 8.6) imply completeness: Let $\Psi \in \mathcal{H}$ and $\varepsilon>0$. Since the spectral projectors of the unperturbed problem converge strongly (i.e. $s-\lim _{L \rightarrow \infty} P_{L}^{0}=1$ ), there is $L \in \mathbb{N}$ such that $\left\|\Psi-P_{M}^{0} \Psi\right\|<\varepsilon$ for all $M \geq L$. According to (8.5), $\left\|P_{K}-\mathbb{1}\right\| \leq$ $\left\|P_{K}-P_{K}^{0}\right\|+\left\|P_{K}^{0}\right\|+\|1\| \leq 3$. Hence for all $K>L$,

$$
\begin{aligned}
\left\|\left(P_{K}-1\right) \Psi\right\| & \leq\left\|\left(P_{K}-1\right)\left(\Psi-P_{L}^{0} \Psi\right)\right\|+\left\|\left(P_{K}-P_{K}^{0}\right) P_{L}^{0} \Psi\right\| \\
& \leq 2 \varepsilon+\left\|\left(P_{K}-P_{K}^{0}\right) P_{L}^{0} \Psi\right\|,
\end{aligned}
$$

and the estimate (8.6) shows that the last term can be made arbitrarily small by choosing $K$ sufficiently large.

It remains to prove the inequalities (1.6) and (1.7). Combining the gap estimates of Theorem 1.3 with the fact that $\mu_{k} \in B_{\rho}\left(\lambda_{N+k}\right)$, one immediately obtains (1.7). The inequality (1.7) follows similarly from the asymptotics (2.9).

Acknowledgments: We would like to thank Joel Smoller and Niky Kamran for helpful discussions, and Johannes Kern for pointing out an error in an earlier version of the proof of Lemma 5.1. We are grateful to the "Centre de Recherche Mathémathiques," Montréal, for support and hospitality. One of us (FF) wants to thank the Max Planck Institute for Mathematics in the Sciences, Leipzig, and the Morningside Center, Beijing, for hospitality and support. Finally, we would like to thank the referee for helpful comments.

\section{References}

[1] M. S. Ashbaugh, The universal eigenvalue bounds of Payne-Pólya-Weinberger, HileProtter, and H.C. Yang, Proc. Indian Acad. Sci. Math. Sci. 112 (2002), no. 1, 3-30.

[2] E.A. Coddington and N. Levinson, The Theory of Ordinary Differential Equations, McGraw Hill (1955)

[3] N. Dunford and J.T. Schwartz, Linear Operators, Part II, Interscience Publishers, John Wiley (1963)

[4] F. Finster, N. Kamran, J. Smoller, S. T. Yau, An Integral Spectral Representation of the Propagator for the Wave Equation in the Kerr Geometry, gr-qc/0310024, Commun. Math. Phys. 260 (2005), 257-298.

[5] C. Flammer, Spheroidal wave functions, Stanford University Press, Stanford, California (1957). 
[6] N. Fröman, P.O. Fröman, JWKB Approximation, North-Holland, Amsterdam (1965)

[7] C. Hunter And B. Guerrieri, The Eigenvalues of the Angular Spheroidal Wave Equation, Stud. Appl. Math. 66 (1982), 217-240.

[8] T. Kato, Perturbation theory for linear operators, 2nd edition, Springer (1980).

[9] R. Lavine, The eigenvalue gap for one-dimensional convex potentials, Proc. Amer. Math. Soc. 121 (1994), no. 3, 815-821.

[10] C.K. LAW And Y.-L. HuAng, Eigenvalue ratios and eigenvalue gaps of SturmLiouville operators, Proc. Roy. Soc. Edinburgh Sect. A 128 (1998), 337-347.

[11] J. MeIXner And F. W. SchäFKe, Mathieusche Funktionen und Sphäroidfunktionen, Die Grundlehren der mathematischen Wissenschaften in Einzeldarstellungen, Band LXXI, Springer (1954).

[12] M. Reed And B. Simon, Methods of Modern Mathematical Physics, IV. Analysis of Operators, Academic Press, New York (1978).

[13] M.E. TaYlor, Partial Differential Equations II, Springer (1996).

[14] I.M. Singer, B. Wong, S.-T. Yau, S. S.-T. Yau, An estimate of the gap of the first two eigenvalues of the Schrödinger operator, Ann. Scuola Norm. Sup. Pisa Sci. (4) 12 (1985).

[15] J. Weidmann, Spectral Theory of Ordinary Differential Operators, Lect. Notes in Math. 1258, Springer (1980).

NWF I - Mathematik, Universität Regensburg, 93040 Regensburg, Germany, Felix.Finster@mathematik.uni-regensburg.de, Harald.Schmid@mathematik. uni-regensburg. de 